\title{
STAR FORMATION RELATIONS AND CO SPECTRAL LINE ENERGY DISTRIBUTIONS ACROSS THE $J$-LADDER AND REDSHIFT
}

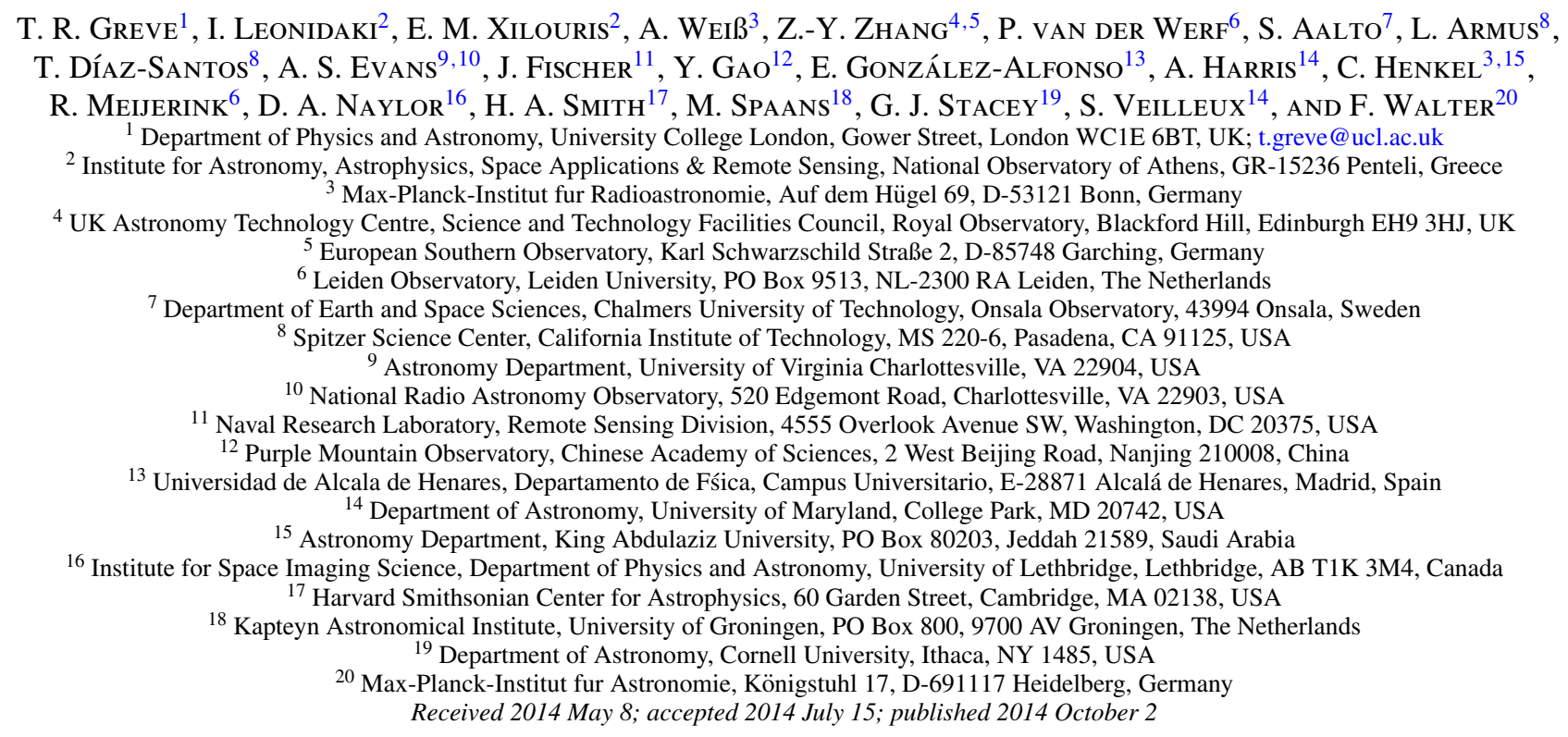

\section{ABSTRACT}

We present FIR [50-300 $\mu \mathrm{m}$ ] $-\mathrm{CO}$ luminosity relations (i.e., $\log L_{\mathrm{FIR}}=\alpha \log L_{\mathrm{CO}}^{\prime}+\beta$ ) for the full CO rotational ladder from $J=1-0$ up to $J=13-12$ for a sample of 62 local $(z \leqslant 0.1)$ (Ultra) Luminous Infrared Galaxies (LIRGs; $\left.L_{\text {IR }[8-1000 \mu \mathrm{m}]}>10^{11} L_{\odot}\right)$ using data from Herschel SPIRE-FTS and ground-based telescopes. We extend our sample to high redshifts $(z>1)$ by including 35 submillimeter selected dusty star forming galaxies from the literature with robust $\mathrm{CO}$ observations, and sufficiently well-sampled FIR/submillimeter spectral energy distributions (SEDs), so that accurate FIR luminosities can be determined. The addition of luminous starbursts at high redshifts enlarge the range of the FIR-CO luminosity relations toward the high-IR-luminosity end, while also significantly increasing the small amount of mid- $J /$ high- $J$ CO line data $(J=5-4$ and higher) that was available prior to Herschel. This new data set (both in terms of IR luminosity and $J$-ladder) reveals linear FIR-CO luminosity relations (i.e., $\alpha \simeq 1$ ) for $J=1-0$ up to $J=5-4$, with a nearly constant normalization $(\beta \sim 2)$. In the simplest physical scenario, this is expected from the (also) linear FIR-(molecular line) relations recently found for the dense gas tracer lines (HCN and CS), as long as the dense gas mass fraction does not vary strongly within our (merger/starburst)-dominated sample. However, from $J=6-5$ and up to the $J=13-12$ transition, we find an increasingly sublinear slope and higher normalization constant with increasing $J$. We argue that these are caused by a warm $(\sim 100 \mathrm{~K})$ and dense $\left(>10^{4} \mathrm{~cm}^{-3}\right.$ ) gas component whose thermal state is unlikely to be maintained by star-formation-powered far-UV radiation fields (and thus is no longer directly tied to the star formation rate). We suggest that mechanical heating (e.g., supernova-driven turbulence and shocks), and not cosmic rays, is the more likely source of energy for this component. The global CO spectral line energy distributions, which remain highly excited from $J=6-5$ up to $J=13-12$, are found to be a generic feature of the (U)LIRGs in our sample, and further support the presence of this gas component.

Key words: galaxies: evolution - galaxies: formation - galaxies: ISM - galaxies: starburst - ISM: molecules

Online-only material: color figures

\section{INTRODUCTION}

Early empirical correlations between the preponderance of young stars and gas in galaxies (e.g., Sanduleak 1969) confirmed-in a qualitative sense- the simple power-law dependence between the star formation rate surface density $\left(\Sigma_{\mathrm{SFR}}\right)$ and gas surface density $\left(\Sigma_{\text {gas }}\right)$ first suggested by Schmidt (1959), who found $\Sigma_{\mathrm{SFR}} \propto \Sigma_{\text {gas }}^{2}$ for $\mathrm{H}$ I gas. Once the $\mathrm{H}_{2}$ component as traced by $\mathrm{CO}$ lines was identified in galaxies, the gas surface density could be related to both $\mathrm{H}_{\mathrm{I}}$ and $\mathrm{H}_{2}$ (i.e., $\Sigma_{\text {gas }}=\Sigma_{\mathrm{H}_{\mathrm{I}}}+\Sigma_{\mathrm{H}_{2}}$;
Kennicutt 1989). In a seminal paper, Kennicutt (1998) established this relation, hereafter called the Schmidt-Kennicutt (S-K) relation, to be $\Sigma_{\mathrm{SFR}} \propto \Sigma_{\text {gas }}^{1.4}$, averaged over entire galaxy disks. Further studies by Wong \& Blitz (2002) and Schruba et al. (2011) found a nearly linear S-K relation for the molecular gas on kpc scales (see also Bigiel et al. 2008 and Leroy et al. 2008, 2013), with the SFR surface density having a much closer correspondence with the molecular gas surface density-reflecting the well-established fact that stars form out of molecular rather than atomic gas. Much theoretical effort has gone into obtaining 
the exponents and normalization of this relation as unique outcomes of various physical processes occurring in star forming galaxies, with various models capable of yielding (S-K)-type relations (e.g., Dopita \& Ryder 1994; Gerritsen 1997; Wong \& Blitz 2002; Elmegreen 2002). It became evident that, while no deterministic microphysics of the interstellar medium (ISM) and star formation (SF) can be linked to a given S-K relation, the high-density gas component $\left(n \geqslant 10^{4} \mathrm{~cm}^{-3}\right)$ plays a crucial role in ultimately anchoring such relations to the star formation taking place deep inside supersonically turbulent molecular clouds in disks.

The S-K relations for high-density gas are particularly challenging to establish because determining the dense gas-mass fraction within a galaxy requires observations of $\mathrm{CO}$ from $J=1-0$ (a total molecular gas mass tracer) up to at least $J=3-2$ along with the much fainter lines of bona fide dense gas tracers, like CS, and heavy-rotor molecules, such as $\mathrm{HCN}$. A multi-component analysis of such $\mathrm{CO}, \mathrm{HCN}$, and CS spectral line energy distributions (SLEDs) can then yield dense gas masses, $M_{\text {dense }}\left(n \geqslant 10^{4} \mathrm{~cm}^{-3}\right.$ ) (e.g., Mao et al. 2000; Greve et al. 2009). However, doing so for a large number of galaxies in order to obtain even a surface-integrated SFR $-M_{\text {dense }} \mathrm{S}-\mathrm{K}$ relation has been prohibitively expensive in telescope time. At high redshifts the situation is made worse due to a lack in sensitivity and angular resolution. Nonetheless, pioneering efforts have been made in discerning $\Sigma_{\mathrm{SFR}}=A \Sigma_{\text {gas }}^{N}$ at high redshifts using $\mathrm{H} \alpha$ maps obtained with integral field unit cameras, and high-resolution interferometric $\mathrm{CO}(J=1-0$ to $3-2)$ observations of massive star forming galaxies at $z \sim 1-3$ (Genzel et al. 2010; Tacconi et al. 2013; Freundlich et al. 2013). This situation will now improve dramatically with the advent of the Atacama Large Millimeter/Submillimeter Array (ALMA).

With the dense gas-mass fraction distribution currently inaccessible for any statistically significant number of galaxies, one must fall back to the integrated (S-K)-proxy relations: $L_{\mathrm{IR}}-L_{\text {line }}$ (where $L_{\text {line }}$ is the line luminosity of a dense gas tracer and $L_{\mathrm{IR}}$ a linear proxy of SFR), and then invoke theoretically determined links to an underlying S-K relation (Krumholz \& Thompson 2007; Narayanan et al. 2008; Narayanan \& Krumholz 2014). HCN(1-0) observations of statistically significant samples of local IR luminous galaxies (LIRGs) and normal spiral galaxies yielded the first of such $(\mathrm{S}-\mathrm{K})$-proxy relations using gas tracers other than CO lines (Solomon et al. 1992), finding the IR $-\mathrm{HCN}$ relation to be linear and with much less scatter than the previously determined IR-CO low- $J$ relations. This was interpreted as $\mathrm{HCN}(1-0)$, with its high critical density $\left(\sim 10^{5} \mathrm{~cm}^{-3}\right)$, being a more direct tracer of a dense, star forming gas component with a nearly constant underlying star formation efficiency (SFE; Gao \& Solomon 2004a, 2004b). Furthermore, with the tight, linear IR-HCN relation extending down to individual Galactic molecular clouds where $L_{\mathrm{IR}} \gtrsim 10^{4.5} L_{\odot}$, thus covering over $\sim 8$ orders of magnitude in luminosity, its origin could be attributed to the existence of fundamental units of cluster star formation (Wu et al. 2005). This view is now further supported by the linear $L_{\mathrm{IR}}-L_{\text {line }}$ relations found for the $\mathrm{HCN}(4-3)$ and CS(7-6) lines (Zhang et al. 2014), which for $\mathrm{CS}(7-6)$ also extends (linearly) down to Galactic cores (Wu et al. 2010). Some contentious points do remain, however, especially toward the high- $L_{\mathrm{IR}}$ end that is dominated by mergers/ starbursts where a slightly super-linear IR-HCN relation has been claimed and argued to be due mostly to an increase in the dense gas SFE in such galaxies (Riechers et al. 2007; GraciáCarpio et al. 2008b).
In this paper we present the first FIR-CO luminosity relations and the corresponding global CO SLEDs that extend above $J_{\text {up }}=4$ and up to $J_{\text {up }}=13$ using Herschel SPIRE-FTS data for local (U)LIRGs. The FIR-CO relations and CO SLEDs presented in this work (from $J=1-0$ up to $J=13-12$ ), besides a significant extension of the $J$-ladder, benefit from the inclusion of (U)LIRGs from the low- and the high- $z$ universe. This robustly extends the sample toward the important high$L_{\text {FIR }}$ end (as numerous galaxies with ULIRG-like, or higher, luminosities have been found in the high- $z$ universe), where very different conditions may prevail for the molecular gas, possibly leaving an imprint on the FIR-CO relations and the $\mathrm{CO}$ SLEDs. Our new high-J CO line data set is uniquely sensitive to such an imprint because these lines need both high densities $\left(n_{\text {crit }} \sim\left(10^{4}-7 \times 10^{5}\right) \mathrm{cm}^{-3}\right)$ and (in most circumstances) high temperatures $\left(E_{J} / k_{\mathrm{B}} \sim(55-500) \mathrm{K}\right)$ to be significantly excited. The high-density and warm gas necessary for exciting them is the most difficult phase to maintain energetically in appreciable quantities in galaxies. However, it is one that would leave no easily discernible signature in the low- $J$ CO and low-/mid- $J$ SLEDs of dense gas tracers (e.g., HCN, CS) that typically have been available for (U)LIRGs up to now. Throughout, we adopt a flat cosmology with $\Omega_{\mathrm{M}}=0.315, \Omega_{\Lambda}=0.685$, and $h=0.67$ (Planck 2013).

\section{GALAXY SAMPLES AND DATA}

For the purposes of this work, we first compiled high- $J$ ( $J=4-3$ up to $J=13-12$ line data from the Herschel Comprehensive (U)LIRG Emission Survey (HerCULES; van der Werf et al. 2010), which is an open time key program on the ESA Herschel Space Observatory. ${ }^{21}$ (Pilbratt et al. 2010) It measured $\mathrm{CO} J=4-3$ to $J=13-12$ for 29 local $(z<0.1)$ (U)LIRGs using the Fourier Transform Spectrometer (FTS) of the SPIRE instrument ${ }^{22}$ (Griffin et al. 2010). The HerCULES sources were selected from the $60 \mu \mathrm{m}$ flux-limited IRAS Revised Bright Galaxy Sample $\left(f_{60 \mu \mathrm{m}}>5.24 \mathrm{Jy}\right.$; Sanders et al. 2003) with separate flux cuts applied to ULIRGs and LIRGs $\left(f_{60 \mu \mathrm{m}}>11.65 \mathrm{Jy}\right.$ and $>16.4 \mathrm{Jy}$, respectively). A detailed description of the SPIRE-FTS observations, calibration modes, extraction of CO line fluxes, and final line luminosities, are given in a dedicated paper (Rosenberg et al., 2014). Briefly, the high spectral resolution mode was used with a resolution of 1.2 $\mathrm{GHz}$ over both observing bands. A reference measurement was used to subtract the emission from the sky, telescope, and instrument. The spectra were reduced using the Herschel Interactive Processing Environment (HIPE), ver. 9.0. At the time of writing, fully reduced SPIRE-FTS CO spectra were available for only 26 sources, and of these 3 had extended, multi-component morphologies and were discarded. Because the SPIRE-FTS beam ranges from $\sim 16^{\prime \prime}$ to $\sim 42^{\prime \prime}$ (FWHM) across the bandpass (Makiwa et al. 2013), it is essential to perform a beam correction in cases where the sources are extended with respect to the beam. All spectra (and thus CO line fluxes) were scaled to a

\footnotetext{
21 Herschel is an ESA space observatory with science instruments provided by European-led Principal Investigator consortia and with important participation from NASA.

22 SPIRE has been developed by a consortium of institutes led by Cardiff Univ. (UK) and including Univ. Lethbridge (Canada); NAOC (China); CEA, LAM (France); IFSI, Univ. Padua (Italy); IAC (Spain); Stockholm Observatory (Sweden); Imperial College London, RAL, UCL-MSSL, UKATC, Univ. Sussex (UK); and Caltech, JPL, NHSC, Univ. Colorado (USA). This development has been supported by national funding agencies, including CSA (Canada); NAOC (China); CEA, CNES, CNRS (France); ASI (Italy); MCINN (Spain); SNSB (Sweden); STFC, UKSA (UK); and NASA (USA).
} 
common spatial resolution of $\sim 42^{\prime \prime}$ using LABOCA $870 \mu \mathrm{m}$ or SABOCA $350 \mu \mathrm{m}$ maps (see Rosenberg et al. 2014 for details). This assumes that the corrections are perfectly mono-chromatic in the FIR and submillimeter (submm) regime, which is a good assumption to within $\lesssim 20 \%$ (Galametz et al. 2013). For the HerCULES sources, which are all (U)LIRGs and thus nearly all relatively compact and well within the beam sizes of the $\mathrm{CO}$ observations, this correction was minor. For very extended sources, however, this correction is crucial, and failing to apply it can skew the observed FIR-CO relation (i.e., Bussmann et al. 2008, Juneau et al. 2009, and see discussion in Zhang et al. 2014).

We also included ground-based CO line data presented by Papadopoulos et al. (2012) for a sample of 45 local (U)LIRGs ${ }^{23}$ from the IRAS RBGS. These data consisted of low-J CO transitions - that is, $J=1-0$ (all 45 sources), 2-1 (17), 3-2 (44)-as well as $J=4-3$ (3) and 6-5 (12) observations. This allowed us to fill in the $J=1-0,2-1,3-2$ transitions for the 11 HerCULES sources that overlapped with this sample (except for 1 source that did not have $J=2-1$ measurement), and bring in additional CO low- $J$ and $J=4-3 / 6-5$ lines (the only mid-/high-J CO lines accessible with the ground-based telescopes used; see Papadopoulos et al. 2012 for details) to the sample. We stress that the $\mathrm{CO}$ line fluxes given in Papadopoulos et al. (2012) are total line fluxes, and so no additional beam correction is required for these sources.

Of our sample of 68 local (U)LIRGs (listed in Table 1), 30 sources $(20+10$ from HerCULES and Papadopoulos et al. 2012 subsamples, respectively) are also part of The Great Observatories All-Sky LIRG Survey (GOALS; Armus et al. 2009). To weed out active galactic nuclei (AGNs), the sample was cross-correlated against estimates of the AGN contribution to the bolometric luminosity based on several MIR diagnostics, such as the equivalent width of the $6.2 \mu \mathrm{m}$ PAH feature, the $[\mathrm{Ne} \mathrm{V}] /[\mathrm{Ne} \mathrm{II}]$ and $\left[\mathrm{O}_{\mathrm{IV}}\right] /[\mathrm{Ne} \mathrm{II}]$ emission line ratios, as well as 30-to-15 $\mu \mathrm{m}$ continuum flux ratios (Veilleux et al. 2009; Petric et al. 2011; Stierwalt et al. 2013, 2014; Inami etal. 2013). Only six sources (indicated by a $*$ in Table 1 ) were found to have an AGN contribution $>30 \%$ and were omitted from our analysis (although, including them in our analysis did not alter the findings of this paper).

The FIR/submm continuum data were obtained from a number of studies (see Papadopoulos et al. 2012 and references therein) as well as from the NASA/IPAC Extragalactic Database (NED). All the $850 \mu \mathrm{m}$ and $1.2 \mathrm{~mm}$ available fluxes were corrected for CO $J=3-2$, and 2-1 line contamination $(<20 \%)$, respectively. We also corrected for any nonthermal radio continuum contributions whenever radio data were available, allowing for a power-law extrapolation to the submm wavelengths. The FIR (50-300 $\mu \mathrm{m})$ luminosities derived from the continuum data (see Section 3 for details) span the range $\sim 10^{10-12} L_{\odot}$ (Figure 1). The two samples are well matched in luminosity, although no HerCULES sources are found at $\lesssim 10^{10.8} L_{\odot}$. The fact that the more luminous sources tend to have higher redshifts merely reflects the flux-limited selection of the two samples.

High-redshift dusty star forming galaxies (DSFGs ${ }^{24}$ ) are thought to resemble the local (U)LIRG population and most

\footnotetext{
23 The full sample in Papadopoulos et al. (2012) consisted of 70 (U)LIRGs, but 25 of those lacked adequate continuum FIR and/or submm data and were discarded.

${ }^{24}$ In this paper we take DSFGs to be synonymous with highly

dust-enshrouded major merger starbursts selected at submm $/ \mathrm{mm}$ wavelengths (also often referred to as (sub)millimeter selected galaxies, i.e., SMGs).
}

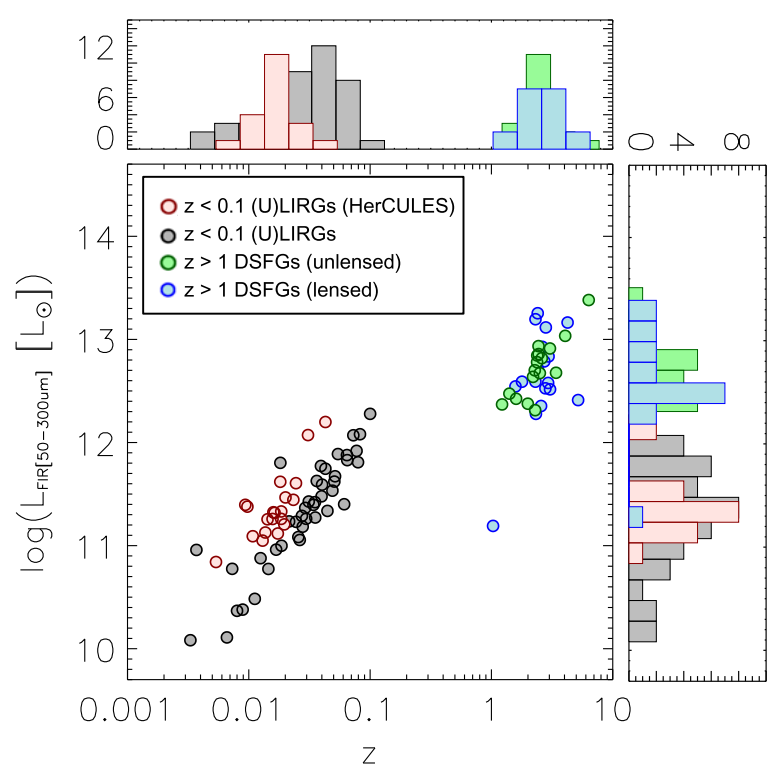

Figure 1. Logarithm of the FIR (50-300 $\mu \mathrm{m})$ luminosity vs. redshift for the galaxy samples considered in this paper (after AGN-dominated systems have been removed), along with histograms of the FIR luminosity and redshift distributions (top and right inserts, respectively). The local $(z<0.1)$ sources include subsets of the (U)LIRG samples from HerCULES (20 sources, red symbols) and Papadopoulos et al. (2012) (42 sources, gray symbols). The high$z(z>1)$ sources are not lensed, or weakly lensed, DSFGs (16 sources, green symbols) and strongly lensed DSFGs (19 sources, blue symbols) uncovered from various submm surveys (see Section 2). All FIR luminosities have been corrected for lensing using the magnification factors in Table 2.

(A color version of this figure is available in the online journal.)

have $L_{\mathrm{IR}} \gtrsim 10^{12} L_{\odot}$. Moreover, multiple high- $J \mathrm{CO}$ lines and $\mathrm{FIR} /(\mathrm{sub}) \mathrm{mm}$ continuum observations are typically available for them. These were our main reasons for including them in the analysis. In order to achieve the best possible uniformity, a meticulous compilation of the aforementioned observations (CO line and continuum observations) for all published DSFGs was extracted from the literature (guided by major review papers by Solomon \& Vanden Bout 2005 and Carilli \& Walter 2013 as well as published CO surveys of large samples of DSFGs by Greve et al. 2005 and Bothwell et al. 2013a). Sources with clear signs of AGNs (e.g., from optical spectroscopy showing strong $\mathrm{Ly} \alpha, \mathrm{C}$ IV, and $\mathrm{C}$ III emission lines and a powerlaw continuum, or radio-loudness) were not included in our sample. In cases where multiple observations of the same $\mathrm{CO}$ transition existed, we adopted the weighted mean of the velocity-integrated line flux after discarding any outliers and measurements with low signal-to-noise. Many of the high- $z$ $\mathrm{CO}$ detections are of strongly lensed DSFGs, which we take to mean a gravitational magnification factor $(\mu)>1$, and in those cases we adopted the best estimates of $\mu$ available at the time of writing (e.g., Swinbank et al. 2010; Aravena et al. 2013; Bussmann et al. 2013). A total of 74 DSFGs comprised our initial high- $z$ sample. For 39 (53\%) of the DSFGs, however, we were unable to put reliable constraints on their FIR luminosities (see Section 3), and were therefore discarded for the analysis presented in this paper. This left us with a final sample of 35 high- $z$ sources (listed in Table 2), spanning the redshift range $z=1.0-6.3$ with a median redshift of $z \simeq 2.4$ (see also Figure 1). The lensed DSFGs (19 in total), after magnification correction, span a similar range in FIR luminosity as the nonlensed DSFGs $\left(\sim 10^{12-14} L_{\odot}\right.$; see Figure 1$)$, which is about an order of magnitude higher than that of the local samples. 
Table 1

The Sample of 68 Local $(z<0.1)$ (U)LIRGs used in this Paper

\begin{tabular}{|c|c|c|c|}
\hline ID & $z$ & $\begin{array}{c}\log \\
\left(L_{\mathrm{FIR}[50-300 \mu \mathrm{m}]} / L_{\odot}\right)\end{array}$ & $\begin{array}{c}\log \\
\left(L_{\mathrm{IR}[8-1000 \mu \mathrm{m}]} / L_{\odot}\right)\end{array}$ \\
\hline IRAS 00085-1223 (NGC 34) & 0.0196 & 11.21 & 11.47 \\
\hline IRAS 00506+7248 (MCG+12-02-001) & 0.0157 & 11.26 & 11.53 \\
\hline IRAS 01053-1746 (IC 1623) & 0.0201 & 11.47 & 11.74 \\
\hline IRAS 04315-0840 (NGC 1614) & 0.0159 & 11.32 & 11.59 \\
\hline IRAS 05189-2524* & 0.0426 & 11.73 & 12.12 \\
\hline IRAS 08354+2555 (NGC 2623) & 0.0185 & 11.33 & 11.60 \\
\hline IRAS 10257-4339 (NGC 3256) & 0.0094 & 11.40 & 11.60 \\
\hline IRAS 11506-3851 (ESO 320-G030) & 0.0108 & 11.09 & 11.30 \\
\hline IRAS $12540+5708($ Mrk 231)* & 0.0422 & 12.14 & 12.56 \\
\hline IRAS $13120-5453$ (WKK 2031) & 0.0308 & 12.07 & 12.34 \\
\hline IRAS $13183+3423$ (Arp 193) & 0.0233 & 11.44 & 11.68 \\
\hline IRAS 13229-2934 (NGC 5135) & 0.0137 & 11.13 & 11.33 \\
\hline IRAS $13242-5713$ (ESO 173-G015) & 0.0097 & 11.38 & 11.65 \\
\hline IRAS $13428+5608$ (Mrk 273)* & 0.0378 & 11.91 & 12.17 \\
\hline IRAS 16504+0228 (NGC 6240) & 0.0245 & 11.61 & 11.87 \\
\hline IRAS $15107+0724$ (Zw 049.057) & 0.0130 & 11.05 & 11.28 \\
\hline IRAS $17208-0014$ & 0.0428 & 12.20 & 12.47 \\
\hline IRAS 18093-5744 (IC 4687) & 0.0173 & 11.12 & 11.39 \\
\hline IRAS $18293-3413$ & 0.0182 & 11.62 & 11.84 \\
\hline IRAS 23007+0836 (NGC 7469) & 0.0163 & 11.32 & 11.60 \\
\hline IRAS 23134-4251 (NGC 7552) & 0.0054 & 10.84 & 11.05 \\
\hline IRAS $23488+2018$ (Mrk 331) & 0.0185 & 11.26 & 11.53 \\
\hline IRAS 23488+1949 (NGC 7771) & 0.0143 & 11.26 & 11.43 \\
\hline IRAS $00057+4021$ & 0.0445 & 11.34 & 11.60 \\
\hline IRAS $00509+1225$ & 0.0611 & 11.40 & 11.67 \\
\hline IRAS 01077-1707 & 0.0351 & 11.42 & 11.69 \\
\hline IRAS $01418+1651$ & 0.0274 & 11.29 & 11.56 \\
\hline IRAS $02114+0456$ & 0.0297 & 11.26 & 11.43 \\
\hline IRAS 02401-0013 & 0.0037 & 10.96 & 11.23 \\
\hline IRAS $02483+4302$ & 0.0514 & 11.67 & 11.85 \\
\hline IRAS $02512+1446$ & 0.0312 & 11.43 & 11.70 \\
\hline IRAS $03359+1523$ & 0.0353 & 11.27 & 11.45 \\
\hline IRAS $04232+1436$ & 0.0795 & 11.81 & 12.08 \\
\hline IRAS $05083+7936$ & 0.0543 & 11.88 & 12.06 \\
\hline IRAS $08572+3915 *$ & 0.0582 & 11.73 & 12.11 \\
\hline IRAS 09126+4432 & 0.0398 & 11.48 & 11.65 \\
\hline IRAS $09320+6134$ & 0.0393 & 11.77 & 11.95 \\
\hline IRAS $09586+1600$ & 0.0080 & 10.37 & 10.64 \\
\hline IRAS $10035-4852$ & 0.0648 & 11.83 & 12.10 \\
\hline IRAS 10039-3338* & 0.0341 & 11.47 & 11.74 \\
\hline IRAS $10173+0828$ & 0.0489 & 11.53 & 11.80 \\
\hline IRAS $10356+5345$ & 0.0033 & 10.08 & 10.35 \\
\hline IRAS $10565+2448$ & 0.0428 & 11.75 & 12.01 \\
\hline IRAS $11231+1456$ & 0.0341 & 11.40 & 11.57 \\
\hline IRAS $12001+0215$ & 0.0066 & 10.11 & 10.29 \\
\hline IRAS $12112+0305$ & 0.0727 & 12.07 & 12.34 \\
\hline IRAS 12224-0624 & 0.0263 & 11.05 & 11.23 \\
\hline IRAS $12243-0036$ & 0.0073 & 10.77 & 11.04 \\
\hline IRAS 13001-2339 & 0.0215 & 11.24 & 11.41 \\
\hline IRAS $13102+1251$ & 0.0112 & 10.48 & 10.66 \\
\hline IRAS $13188+0036$ & 0.0186 & 11.00 & 11.17 \\
\hline IRAS $13362+4831$ & 0.0278 & 11.18 & 11.45 \\
\hline IRAS $13470+3530$ & 0.0168 & 10.96 & 11.10 \\
\hline IRAS $13564+3741$ & 0.0125 & 10.88 & 11.05 \\
\hline IRAS $14003+3245$ & 0.0145 & 10.77 & 10.95 \\
\hline IRAS $14178+4927$ & 0.0256 & 11.08 & 11.35 \\
\hline IRAS $14348-1447$ & 0.0825 & 12.08 & 12.42 \\
\hline IRAS $15163+4255$ & 0.0402 & 11.59 & 11.94 \\
\hline IRAS $15243+4150$ & 0.0089 & 10.38 & 10.65 \\
\hline IRAS $15327+2340$ & 0.0182 & 11.80 & 11.98 \\
\hline IRAS 15437+0234* & 0.0128 & 10.84 & 11.01 \\
\hline IRAS 16104+5235 & 0.0292 & 11.37 & 11.63 \\
\hline IRAS $16284+0411$ & 0.0245 & 11.23 & 11.40 \\
\hline IRAS $17132+5313$ & 0.0507 & 11.62 & 11.89 \\
\hline
\end{tabular}


Table 1

(Continued)

\begin{tabular}{lccc}
\hline \hline ID & $z$ & $\log$ & $\log$ \\
& & $\left(L_{\mathrm{FIR}[50-300 \mu \mathrm{m}]} / L_{\odot}\right)$ & $\left(L_{\mathrm{IR}[8-1000 \mu \mathrm{m}]} / L_{\odot}\right)$ \\
\hline IRAS 19458+0944 & 0.1000 & 12.28 & 12.45 \\
IRAS 20550+1656 & 0.0363 & 11.63 & 11.97 \\
IRAS 22491-1808 & 0.0773 & 11.92 & 12.19 \\
IRAS 23365+3604 & 0.0644 & 11.88 & 12.15 \\
\hline
\end{tabular}

Notes. The first 23 sources listed below (and not listed in italics) were observed by Herschel/SPIRE-FTS as part of the HerCULES program (Section 2). Sources indicated by a $*$ were found to have significant AGN contribution ( $>30 \%$ of the bolometric luminosity) and were not included in our final analysis.

Table 2

The High- $z$ DSFGs Samples Utilized in this Paper, Consisting of 35 Sources in Total, of which 19 are Strongly Lensed, i.e., Gravitational Magnification factor $\mu>1$ (Bottom 19, Shown in Italics)

\begin{tabular}{|c|c|c|c|c|c|}
\hline ID & $z$ & $\begin{array}{c}\log \\
\left(L_{\mathrm{FIR}} / L_{\odot}\right)\end{array}$ & $\begin{array}{c}\log \\
\left(L_{\mathrm{IR}} / L_{\odot}\right)\end{array}$ & $\mu$ & Ref. \\
\hline SMM J021725-045934 (SXDF 11) & 2.2920 & 12.30 & 12.51 & 1.0 & {$[1,2]$} \\
\hline SMM J030227.73+000653.3 & 1.4060 & 12.45 & 12.72 & 1.0 & [1] \\
\hline SMM J105151.69+572636.0 (Lock850.16) & 1.5973 & 12.40 & 12.67 & 1.0 & [1] \\
\hline SMM J105227.58+572512.4 (LE 1100.16) & 2.4432 & 12.92 & 13.26 & 1.0 & {$[1]$} \\
\hline SMM J105230.73+572209.5 (LE 1100.05) & 2.6011 & 12.80 & 13.08 & 1.0 & {$[1,3]$} \\
\hline SMM J105238.30+572435.8 (LE 1100.08) & 3.0360 & 12.90 & 13.17 & 1.0 & {$[3]$} \\
\hline SMM J123549.44+621536.8 (AzGN 15, HDF 76) & 2.2020 & 12.62 & 12.89 & 1.0 & {$[1,4]$} \\
\hline SMM J123600.16+621047.3 & 1.9941 & 12.36 & 12.53 & 1.0 & {$[1,3]$} \\
\hline SMM J123606.85+621047.2 & 2.5054 & 12.66 & 12.83 & 1.0 & [1] \\
\hline SMM J123634.51+621240.9 (GN 26, HDF 169) & 1.2224 & 12.35 & 12.61 & 1.0 & {$[5,6]$} \\
\hline SMM J123711.86+622212.6 (GN 20, AzGN 01) & 4.0554 & 13.03 & 13.23 & 1.0 & {$[7,8]$} \\
\hline SMM J131201.17+424208.1 & 3.4078 & 12.67 & 12.94 & 1.0 & {$[3,6,9]$} \\
\hline SMM J163631.47+405546.9 (N2 850.13) & 2.2767 & 12.69 & 12.96 & 1.0 & {$[1,3]$} \\
\hline SMM J163658.19+410523.8 (N2 850.02) & 2.4546 & 12.84 & 13.11 & 1.0 & {$[3,4,10]$} \\
\hline SMM J163650.43+405734.5 (N2 850.04) & 2.3853 & 12.83 & 13.10 & 1.0 & {$[1,10,11]$} \\
\hline SMM J163706.51+405313.8 (N2 1200.17) & 2.3774 & 12.77 & 12.96 & 1.0 & {$[1,3]$} \\
\hline 1HERMESS250 J022016.5060143 (HХMM01) & 2.3074 & 13.17 & 13.37 & $1.5 \pm 0.3$ & [12] \\
\hline SMM J02399-0136 & 2.8076 & 13.08 & 13.43 & $2.38 \pm 0.08(2.45)$ & {$[13,14]$} \\
\hline SPT-S J053816-5030.8 & 2.7818 & 12.49 & 12.69 & $20 \pm 4$ & {$[15,16]$} \\
\hline HATLAS J084933.4+021443-T & 2.4090 & 12.98 & 13.19 & $2.8 \pm 0.2(1.5 \pm 0.2)$ & {$[17,18]$} \\
\hline HATLAS J084933.4+021443-W & 2.4068 & 13.24 & 13.51 & 1.0 & [17] \\
\hline H-ATLASJ090302.9-014128-17b (SDP.17b) & 2.3051 & 12.01 & 12.21 & $4.9 \pm 0.7(18 \pm 8)$ & {$[19,20,21]$} \\
\hline H-ATLASJ090311.6+003906 (SDP.81) & 3.0425 & 12.15 & 12.35 & $11.1 \pm 1.1(14 \pm 4,18-31)$ & {$[18,20,22]$} \\
\hline H-ATLASJ090740.0-004200 (SDP.9) & 1.5770 & 13.47 & 13.67 & $8.8 \pm 2.2$ & {$[18,20]$} \\
\hline H-ATLASJ091043.1-000322 (SDP.11) & 1.7860 & 13.61 & 13.88 & $10.9 \pm 1.3$ & {$[18,20]$} \\
\hline H-ATLASJ091305.0-005343 (SDP.130) & 2.6256 & 12.46 & 12.66 & $2.1 \pm 0.3(5-7,10 \pm 4)$ & {$[18,20,21,22]$} \\
\hline HERMES J105751.1+573027 (HLSW-01) & 2.9574 & 12.82 & 13.17 & $10.9 \pm 0.7(9.2 \pm 0.4)$ & {$[18,23,24]$} \\
\hline SMM J12365+621226 (HDF 850.1) & 5.1830 & 12.43 & 12.65 & 1.4 & [25] \\
\hline$S M M J 14009+0252$ & 2.9344 & 12.57 & 12.74 & 1.5 & {$[14,26,27]$} \\
\hline SMM J140104.96+025223.5 (SMM J14011+0252) & 2.5653 & 12.19 & 12.39 & $3.5 \pm 0.5(2.75 \pm 0.25)$ & {$[28,29,30]$} \\
\hline H-ATLASJ142413.9+023040 (ID141) & 4.2430 & 13.82 & 14.09 & $4.6 \pm 0.5$ & {$[31,32]$} \\
\hline SMM J163555.2+661150(ABELL 2218 Arc L) & 1.0313 & 11.16 & 11.34 & 7.1 & [33] \\
\hline 1HERMESS350 J170647.8+584623 (HFLS3) & 6.3369 & 13.38 & 13.72 & 1.0 & {$[33,34]$} \\
\hline SMMJ2135-0102 (Eyelash) & 2.3259 & 12.26 & 12.36 & $32.5 \pm 4.5$ & {$[35,36]$} \\
\hline$S P T-S 233227-5358.5$ & 2.7256 & 12.77 & 13.04 & $15 \pm 5$ & [15] \\
\hline
\end{tabular}

Notes. The listed FIR $(50-300 \mu \mathrm{m})$ and IR $(8-1000 \mu \mathrm{m})$ luminosities have not been corrected for gravitational lensing, but we give the most up-to-date estimates of the magnification $(\mu)$ factor (from the literature) needed to perform this correction along with the appropriate references for each source. For completeness and for cross-comparison, we also give alternative, but now most likely outdated, magnification estimates in parentheses.

References. [1] Bothwell et al. 2013b; [2] Alaghband-Zadeh et al. 2013; [3] Greve et al. 2005; [4] Tacconi et al. 2006; [5] Frayer et al. 2008; [6] Engel et al. 2010; [7] Daddi et al. 2009; [8] Hodge et al. 2012; [9] Riechers et al. 2011b; [10] Ivison et al. 2011; [11] Neri et al. 2003; [12] Fu et al. 2013; [13] Ivison et al. 2010; [14] Thomson et al. 2012; [15] Aravena et al. 2013; [16] Bothwell et al. 2013a; [17] Ivison et al. 2013; [18] Bussmann et al. 2013; [19] Bussmann et al. 2013; [20] Lupu et al. 2012; [21] Harris et al. 2012; [22] Frayer et al. 2011; [23] Riechers et al. 2011a; [24] Conley et al. 2011; [25] Walter et al. 2012; [26] Weiß et al. 2009; [27] Harris et al. 2010; [28] Frayer et al. 1999; [29] Downes \& Solomon 2003; [30] Sharon et al. 2013; [31] Cox et al. 2011; [32] Bussmann et al. 2012; [33] Riechers et al. 2013; [34] Robson et al. 2014; [35] Swinbank et al. 2010; [36] Danielson et al. 2011. 
The only exception is SMM J163555.2+661150 $(z=1.03)$, which has an intrinsic luminosity similar to that of local LIRGs (Knudsen et al. 2009). Within the high- $z$ samples, we see no strong dependence of FIR luminosity on redshift, which is due to the well-known flat selection function at submm wavelengths for $z \gtrsim 1$ (Blain \& Longair 1993). Finally, we stress that while the DSFGs, as a sample, cover all CO transitions from $J=1-0$ to $J=10-9$, no individual galaxy has continuous coverage across this transition range.

\section{ANALYSIS \\ 3.1. SED Fitting}

The pan-chromatic (FUV/optical to radio) spectral energy distributions (SEDs) of our sample galaxies were modeled using CIGALE (Code Investigating GALaxy Emission; Burgarella et al. 2005; Noll et al. 2009). CIGALE employs dust-attenuated stellar population models to fit the FUV/optical SED, while at the same time ensuring that the dust-absorbed UV photons are re-emitted in the FIR, thus maintaining energy balance between the FUV and FIR. The FIR/submm continuum is modeled using the templates by Dale \& Helou (2002) and Chary \& Elbaz (2001). The Salpeter initial mass function was used for the stellar emission population synthesis models from Maraston (2005), and for the reddening we used attenuation curves from Calzetti et al. (1994) with a wide range of $V$-band attenuation values for young stellar populations. Despite having carefully checked our samples against AGNs, we allowed for the possibility of additional dust emission from deeply buried AGNs by including the 32 AGN models from the Fritz et al. (2006) library in our SED fits. Reassuringly, the AGN fraction never exceeded $20 \%$ of the total IR luminosity. Excellent fits were obtained for all the local galaxies due to their well-sampled SEDs. For the high- $z$ galaxies, only sources with data points longward and shortward of (or near) the dust peak $\left(\lambda_{\text {rest }} \sim 100 \mu \mathrm{m}\right)$ were included in the final analysis, which was a total of 35 out of the original 74 DSFGs. All SED fits used in this paper can be found at http://demogas.astro.noa.gr, and will also be presented in a forthcoming paper (E. M. Xilouris et al., in preparation).

From the SED fits we derived the IR ( $L_{\mathrm{IR}}$, from $8 \mu \mathrm{m}$ to $1000 \mu \mathrm{m}$ rest-frame), and the FIR ( $L_{\mathrm{FIR}}$, from $50 \mu \mathrm{m}$ to $300 \mu \mathrm{m}$ rest-frame), luminosities of our sample galaxies (Tables 1 and 2). We use the latter for our analysis in order to minimize the effects of AGNs, which are strongest in the mid-IR regime (i.e., $\sim 8-40 \mu \mathrm{m}$ ). In addition, the mid-IR is rich in PAH emission/absorption features, which could affect $L_{\mathrm{IR}}$ estimates. For the uncertainty on our IR/FIR luminosity estimates, we adopted the $1 \sigma$ dispersion of the luminosity distributions obtained through bootstrapping the photometry errors 1000 times. Typical uncertainties, $\delta L_{\mathrm{FIR}}$, were $\sim 20 \%$ and $\sim 40 \%$ for the local and high- $z$ samples, respectively, and were adopted across the board for the two samples. We stress that the above FIR luminosities are total luminosities, that is, derived from aperture fluxes that encompass the full extent of the galaxies, and thus match the $\mathrm{CO}$ measurements.

\section{2. $L_{\mathrm{FIR}}-L_{\mathrm{CO}}^{\prime}$ Relations}

Figure 2 shows the separate $\log L_{\mathrm{FIR}}-\log L_{\mathrm{CO}}^{\prime}$ relations (where $\log$ is for base 10) for each $\mathrm{CO}$ transition (from $\mathrm{CO}$ $J=1-0$ to $J=13-12$ ) for the galaxy samples analyzed here. Highly significant correlations are seen in all transitions, as given by their near unity linear correlation coefficients $(r$; see Figure 2). Even for the highest transition $(J=13-12)$, where
Table 3

Best-Fit Slopes $(\alpha)$ and Intersection Points $(\beta)$, Along with the Associated Scatter of the Data Around the Best-Fit Relation, Inferred from Figure 2

\begin{tabular}{|c|c|c|c|}
\hline Transition & $\alpha$ & $\beta$ & $s$ \\
\hline $\mathrm{CO}(1-0)$ & $\begin{array}{c}0.99 \pm 0.04 \\
(1.00 \pm 0.05)\end{array}$ & $\begin{array}{c}1.9 \pm 0.4 \\
(2.0 \pm 0.5)\end{array}$ & $\begin{array}{c}0.26 \\
(0.27)\end{array}$ \\
\hline $\mathrm{CO}(2-1)$ & $\begin{array}{c}1.03 \pm 0.09 \\
(1.05 \pm 0.10)\end{array}$ & $\begin{array}{c}1.6 \pm 0.9 \\
(1.7 \pm 0.9)\end{array}$ & $\begin{array}{c}0.25 \\
(0.27)\end{array}$ \\
\hline $\mathrm{CO}(3-2)$ & $\begin{array}{c}0.99 \pm 0.04 \\
(1.00 \pm 0.05)\end{array}$ & $\begin{array}{c}2.1 \pm 0.4 \\
(2.2 \pm 0.5)\end{array}$ & $\begin{array}{c}0.26 \\
(0.28)\end{array}$ \\
\hline $\mathrm{CO}(4-3)$ & $\begin{array}{c}1.08 \pm 0.09 \\
(1.08 \pm 0.09)\end{array}$ & $\begin{array}{c}1.2 \pm 0.9 \\
(1.5 \pm 0.9)\end{array}$ & $\begin{array}{c}0.30 \\
(0.29)\end{array}$ \\
\hline $\mathrm{CO}(5-4)$ & $\begin{array}{c}0.97 \pm 0.06 \\
(0.97 \pm 0.06)\end{array}$ & $\begin{array}{c}2.5 \pm 0.6 \\
(2.8 \pm 0.6)\end{array}$ & $\begin{array}{c}0.23 \\
(0.23)\end{array}$ \\
\hline $\mathrm{CO}(6-5)$ & $\begin{array}{c}0.93 \pm 0.05 \\
(0.95 \pm 0.06)\end{array}$ & $\begin{array}{c}3.1 \pm 0.5 \\
(3.2 \pm 0.5)\end{array}$ & $\begin{array}{c}0.17 \\
(0.18)\end{array}$ \\
\hline $\mathrm{CO}(7-6)$ & $\begin{array}{c}0.87 \pm 0.05 \\
(0.87 \pm 0.05)\end{array}$ & $\begin{array}{c}3.9 \pm 0.4 \\
(4.1 \pm 0.4)\end{array}$ & $\begin{array}{c}0.19 \\
(0.19)\end{array}$ \\
\hline $\mathrm{CO}(8-7)$ & $\begin{array}{c}0.66 \pm 0.07 \\
(0.66 \pm 0.07)\end{array}$ & $\begin{array}{c}5.8 \pm 0.6 \\
(6.1 \pm 0.6)\end{array}$ & $\begin{array}{c}0.22 \\
(0.20)\end{array}$ \\
\hline $\mathrm{CO}(9-8)$ & $\begin{array}{c}0.82 \pm 0.07 \\
(0.85 \pm 0.07)\end{array}$ & $\begin{array}{c}4.6 \pm 0.6 \\
(4.6 \pm 0.6)\end{array}$ & $\begin{array}{c}0.24 \\
(0.22)\end{array}$ \\
\hline $\mathrm{CO}(10-9)$ & $\begin{array}{c}0.66 \pm 0.07 \\
(0.69 \pm 0.08)\end{array}$ & $\begin{array}{c}6.1 \pm 0.6 \\
(6.1 \pm 0.6)\end{array}$ & $\begin{array}{c}0.27 \\
(0.27)\end{array}$ \\
\hline $\mathrm{CO}(11-10)$ & $\begin{array}{c}0.57 \pm 0.09 \\
(0.61 \pm 0.09)\end{array}$ & $\begin{array}{c}6.8 \pm 0.7 \\
(6.8 \pm 0.7)\end{array}$ & $\begin{array}{c}0.18 \\
(0.17)\end{array}$ \\
\hline $\mathrm{CO}(12-11)$ & $\begin{array}{c}0.51 \pm 0.11 \\
(0.55 \pm 0.11)\end{array}$ & $\begin{array}{c}7.5 \pm 0.8 \\
(7.5 \pm 0.8)\end{array}$ & $\begin{array}{c}0.23 \\
(0.23)\end{array}$ \\
\hline $\mathrm{CO}(13-12)$ & $\begin{array}{c}0.47 \pm 0.20 \\
(0.51 \pm 0.21)\end{array}$ & $\begin{array}{c}7.9 \pm 1.5 \\
(7.9 \pm 1.6)\end{array}$ & $\begin{array}{c}0.30 \\
(0.31)\end{array}$ \\
\hline
\end{tabular}

Notes. The corresponding values using $L_{\operatorname{IR}[8-1000 \mu \mathrm{m}]}$ instead of $L_{\mathrm{FIR}[50-300 \mu \mathrm{m}]}$ are given in parentheses.

the dynamical range spanned in luminosities is relatively small, we see a statistically significant correlation. To ensure that the observed correlations are not simply due to both $L_{\mathrm{IR}}$ and $L_{\mathrm{CO}}^{\prime}$ being $\propto D_{\mathrm{L}}^{2}$ (the luminosity distance squared), we calculated for each correlation the partial Kendall $\tau$-statistic (Akritas \& Siebert 1996) with $D_{\mathrm{L}}^{2}$ as the test variable. In all cases (up to $J=13-12$ ), we find probabilities $P<10^{-6}$ that the observed FIR-CO correlations are falsely induced by the fact that luminosity $\propto D_{L}^{2}$.

A function of the form $\log L_{\mathrm{FIR}}=\alpha \log L_{\mathrm{CO}}^{\prime}+\beta$ was adopted to model the correlations, and the optimal values of the model parameters ( $\alpha$ and $\beta$ ) were fitted (to this end we used the IDL routine linmix_err; Kelly 2007). The slopes $(\alpha)$ and intersection points $(\beta)$ inferred from fits to the combined lowand high- $z$ samples are given in Table 3, along with the scatter $(s)$ of the data points around the fitted relations. The fits are shown as dashed lines in Figure 2. The best-fit $(\alpha, \beta)$-values obtained by using $L_{\mathrm{IR}}$ instead of $L_{\mathrm{FIR}}$ are also listed in Table 3. Within the errors, the fitted parameters are seen to be robust against the adopted choice of FIR or IR luminosity. Also, our results did not change in any significant way when omitting the lensed DSFGs from the analysis. Often lensing amplification factors are uncertain, and strong lensing cannot only skew the selection of sources toward more compact (and thus more likely warm) sources, but for a given source it may also boost the high$J$ CO lines relative to the lower lines (this is discussed further in Section 6.2).

Figures 3 and 4 show the slopes and normalizations, respectively, of the $\log L_{\mathrm{FIR}}-\log L_{\mathrm{CO}}^{\prime}$ relations derived above as a function of the critical densities probed by the various $\mathrm{CO}$ transitions. The critical densities are calculated as $n_{\text {crit }}=A_{\mathrm{ul}} / \sum_{i \neq u} C_{u i}$, 


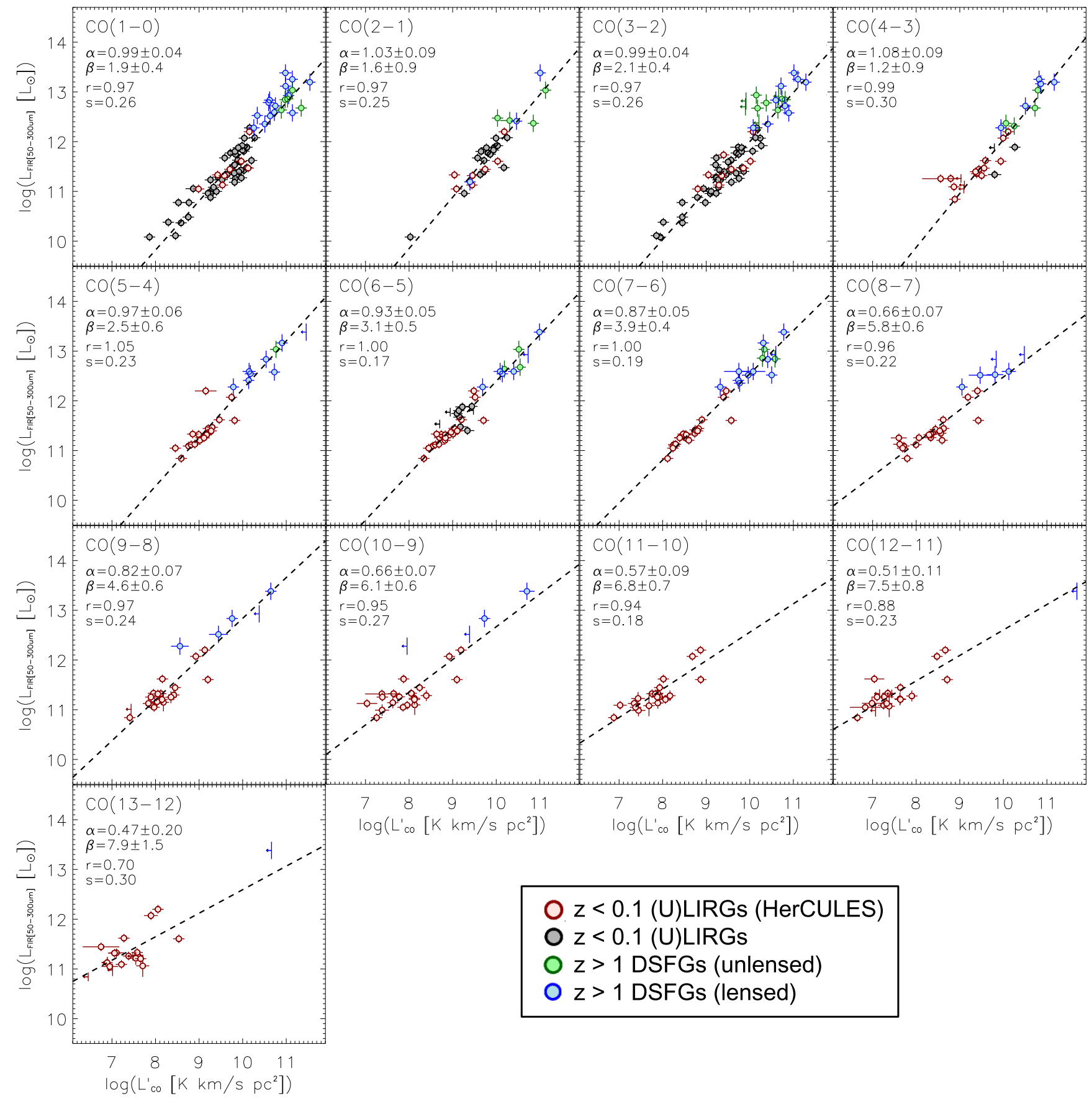

Figure 2. $\log L_{\mathrm{FIR}}$ vs. $\log L_{\mathrm{CO}}^{\prime}$ across the CO rotational ladder (from $J=1-0$ to $\left.J=13-12\right)$. The low- $z(z<0.1)$ data includes the (U)LIRG sample from Papadopoulos et al. (2012; gray symbols) with CO observations from $J=1-0$ to $J=6-5$, and (U)LIRGs from HerCULES (red symbols) observed in CO $J=4-3$ to $J=13-12$ with Herschel SPIRE-FTS. Of the HerCULES sample, 11 sources have CO $J=1-0$ to $J=3-2$ coverage from Papadopoulos et al. (2012; see Section 2). The high- $z(z>1)$ sources are non-lensed DSFGs (green symbols) and strongly lensed $(\mu>1)$ DSFGs (blue symbols) uncovered by various (sub)mm surveys (Section 2). The dashed lines show the best fits of the function $\log L_{\mathrm{FIR}}=\alpha \log L_{\mathrm{CO}}^{\prime}+\beta$ to the data (Section 3), with the optimum parameter $(\alpha, \beta)$ values and their errors indicated in each panel. Also shown in each panel are the correlation coefficients $(r)$ of the data and their scatter $(s)$ around the best-fit line. The $L_{\mathrm{FIR}}$-values used here were obtained by integrating the SEDs across the wavelength range 50-300 $\mu \mathrm{m}$ (Section 3), but near-identical relations are obtained if instead the full IR-luminosity from 8 to $1000 \mu \mathrm{m}$ is used (Table 3). Excluding the lensed DSFGs from the analysis did not significantly alter the best-fit values of $\alpha$ and $\beta$.

(A color version of this figure is available in the online journal.)

where $A_{\mathrm{ul}}$ is the Einstein coefficient for spontaneous decay, and $\sum_{i \neq u} C_{u i}$ is the sum over all collisional coefficients (with $\mathrm{H}_{2}$ as the collisional partner) out of the level $u$, upward and downward (see Table 4 where, as a reference, we also list $n_{\text {crit }}$-values for a number of $\mathrm{HCN}$ and CS transitions). Although it is the first three levels up or down from the $u$-level (i.e., $|u-i|<3$ ) that dominate the sum, often in the literature molecular line crit- ical densities are only calculated for a two-level system (i.e., $|u-i|=1$ ) or for the downward transitions-both practices can significantly overestimate the true $n_{\text {crit }}$ for a given transition. The collision rates were adopted from the Leiden Atomic and Molecular Database (LAMDA; Schöier 2005) for $T_{\mathrm{k}}=40 \mathrm{~K}$, which is within the range of typical dust and gas temperatures encountered in local (U)LIRGs and high-z DSFGs (e.g., Kovács 


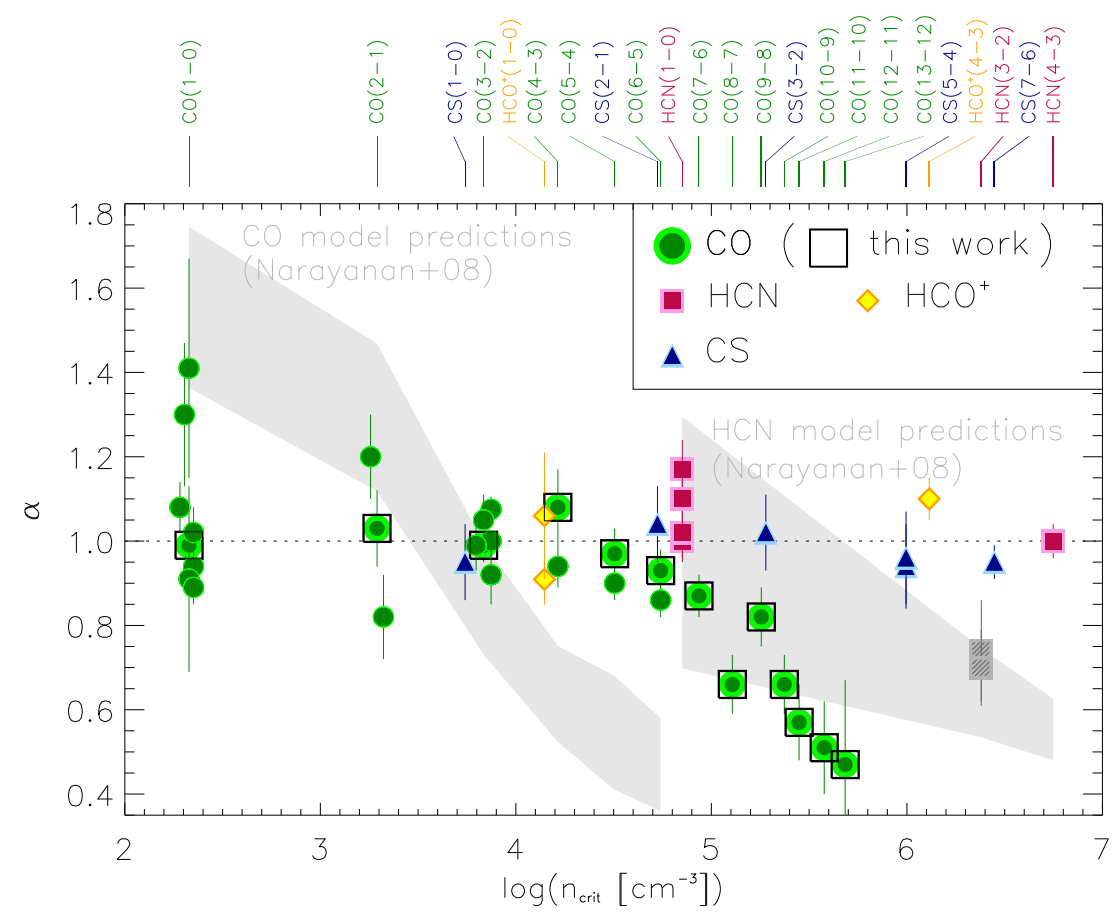

Figure 3. Compilation of slope $(\alpha)$ determinations from the literature for CO (Yao et al. 2003; Gao \& Solomon 2004b; Narayanan et al. 2005; Baan et al. 2008; Juneau et al. 2009; Iono et al. 2009; Bayet et al. 2010; Genzel et al. 2010; Mao et al. 2010), HCO+ (Baan et al. 2008; García-Burillo 2012), HCN (Gao \& Solomon 2004b; Wu et al. 2005, 2010; Bussmann et al. 2008; Graciá-Carpio et al. 2008b; Juneau et al. 2009; García-Burillo 2012; Zhang et al. 2014), and CS (Wu et al. 2010; Wang et al. 2011; Zhang et al. 2014; the CS $J=1-0,2-1,3-2$, and 5-4 results are from Z.-Y. Zhang et al., in preparation). In some cases (Yao et al. 2003; Baan et al. 2008) we had to refit the FIR-CO relations so as to facilitate a direct comparison with our findings (see Section 4.1). The slopes derived in this paper are outlined by black squares. For the first three CO transitions, the different $\alpha$-estimates have been slightly offset horizontally in order to ease the comparison. The gray shaded regions show the $\mathrm{CO}$ (left) and $\mathrm{HCN}$ (right) slopes (within a $1 \sigma$ scatter) predicted by one of two theoretical models (Narayanan et al. 2008). The $L_{\mathrm{FIR}}-L_{\mathrm{X}}^{\prime}$ relations for lines with high $n_{\text {crit }}$ but low $E_{J} / k_{\mathrm{B}}(\mathrm{X}=\mathrm{HCN}, \mathrm{CS})$ have slopes consistent with unity across the critical density regime of $\sim 10^{4-7} \mathrm{~cm}^{-3}$, and are inconsistent with the theoretical predictions. A statistically significant trend of $\alpha$ vs. $n_{\text {crit }}$ is found for the $L_{\mathrm{IR}}-L_{\mathrm{CO}}^{\prime}$ relation with $\alpha \simeq 1$ up to CO $J=5-4$, but then decreasing with higher $J$ (and thus $n_{\text {crit }}$ ). However, the high $E_{J} / k_{\mathrm{B}}$ values of the CO $J=6-5$ to $J=13-12$ transitions $(\sim 115-500 \mathrm{~K})$ place them well outside the applicability of both current theoretical models, and thus this trend cannot be used to test them. Following the reasoning laid out by Krumholz \& Thompson (2007), the sublinear slopes of the $L_{\mathrm{FIR}}-L_{\mathrm{CO}}^{\prime}$ relations for such high- $J \mathrm{CO}$ lines are actually quite unexpected (see discussion). We ignore the FIR-HCN(3-2) slopes inferred by Bussmann et al. (2008) and Juneau et al. (2009; shown as gray hatched squares), because their data were not appropriately beam-corrected (see Section 5.1).

(A color version of this figure is available in the online journal.)

et al. 2006). We do not correct for optical depth effects (i.e., line-trapping) as these are subject to the prevailing average ISM conditions, but we note that large optical depths (especially for low-J CO and HCN lines) can significantly lower the effective critical density to $n_{\text {crit }}^{(\beta)}=\beta_{u l} n_{\text {crit }}$, where $\beta_{u l}$ is the average line escape probability $\left(=\left[1-\exp \left(-\tau_{u l}\right)\right] / \tau_{u l}\right.$ for spherical geometries). The collisional excitation of $\mathrm{CO}$ to higher rotational states $\left(E_{\mathrm{J}}\right)$ is set not only by the gas density but also by its kinetic temperature. The minimum temperature $\left(T_{\min }\right)$ required for significant collisional excitation of a given rotational state is approximately given by $\sim E_{\mathrm{J}} / k_{\mathrm{B}}=B_{\text {rot }} J(J+1) / k_{\mathrm{B}}$, where $B_{\text {rot }}$ is the rotational constant of $\mathrm{CO}$, and $k_{\mathrm{B}}$ is the Boltzmann constant. As a rule of thumb, high kinetic temperatures are needed in order to excite the high- $J$ CO lines (see Table 4), although due to the $n-T_{\mathrm{k}}$ degeneracy, this can also be achieved for very dense, low-temperature gas.

Two trends regarding the $L_{\mathrm{FIR}}-L_{\mathrm{CO}}^{\prime}$ relations become apparent from Figures 3 and 4 (see also Table 3). First, the slopes are linear for $J=1-0$ to $J=5-4$, but then become increasingly sublinear the higher the $J$ level. Second, the normalization parameter $\beta$ remains roughly constant $(\sim 2)$ up to $J=4-3$, $5-4$, but then increases with higher $J$ level, reaching $\beta \sim 8$ for $J=13-12$, which for a given CO luminosity translates into $\sim 6$ orders of magnitude higher $L_{\mathrm{FIR}}$. We stress that although the $L_{\mathrm{FIR}}-L_{\mathrm{CO}}^{\prime}$ relations are linear, and $\beta$ is roughly constant up to
$J=5-4$, it does not generally imply that the CO lines are thermalized (i.e., $L_{\mathrm{CO}_{\mathrm{JJ} J-1}}^{\prime} / L_{\mathrm{CO}_{1.0}}^{\prime} \simeq 1$ ) up to this transition. There is significant scatter within the samples, and while a few sources do have nearly thermalized $J=2-1,3-2$, and/or 4-3 lines, in general, $L_{\mathrm{CO}_{\mathrm{J}, J-1}}^{\prime} / L_{\mathrm{CO}_{1,0}}^{\prime} \lesssim 1$. In fact, rewriting the $L_{\mathrm{FIR}}-L_{\mathrm{CO}}^{\prime}$ relations as

$$
L_{\mathrm{CO}_{\mathrm{J}, \mathrm{J}-1}}^{\prime} / L_{\mathrm{CO}_{1,0}}^{\prime}=L_{\mathrm{FIR}}^{\alpha_{\mathrm{JI}}^{-1}-\alpha_{1,0}^{-1}} \times 10^{\beta_{1,0}-\beta_{J, J-1}},
$$

and inserting the fitted values from Table 3 yields $L_{\mathrm{CO}_{\mathrm{J} J \mathrm{~J}-1}}^{\prime} / L_{\mathrm{CO}_{1,0}}^{\prime}<1$ over the range $L_{\mathrm{FIR}}=10^{9-14} L_{\odot}$.

In the following sections, we discuss these empirical relations in the context of existing theoretical models and the new observational studies of such relations using dense gas tracers like $\mathrm{HCN}$ and $\mathrm{CS}$.

\section{THE SLOPE OF THE $L_{\mathrm{FIR}}-L_{\mathrm{CO}}^{\prime}$ RELATIONS}

\subsection{Comparison with Previous Studies}

Before comparing our derived FIR-CO slopes with those from the literature, we must add two cautionary notes: (1) many studies examine the $L_{\mathrm{CO}}^{\prime}-L_{\mathrm{FIR}}$ relation rather than $L_{\mathrm{FIR}}-L_{\mathrm{CO}}^{\prime}$, and one cannot compare the two simply by inferring the inverse relation; (2) often only the errors in one variable (typically $L_{\mathrm{CO}}^{\prime}$ ) 


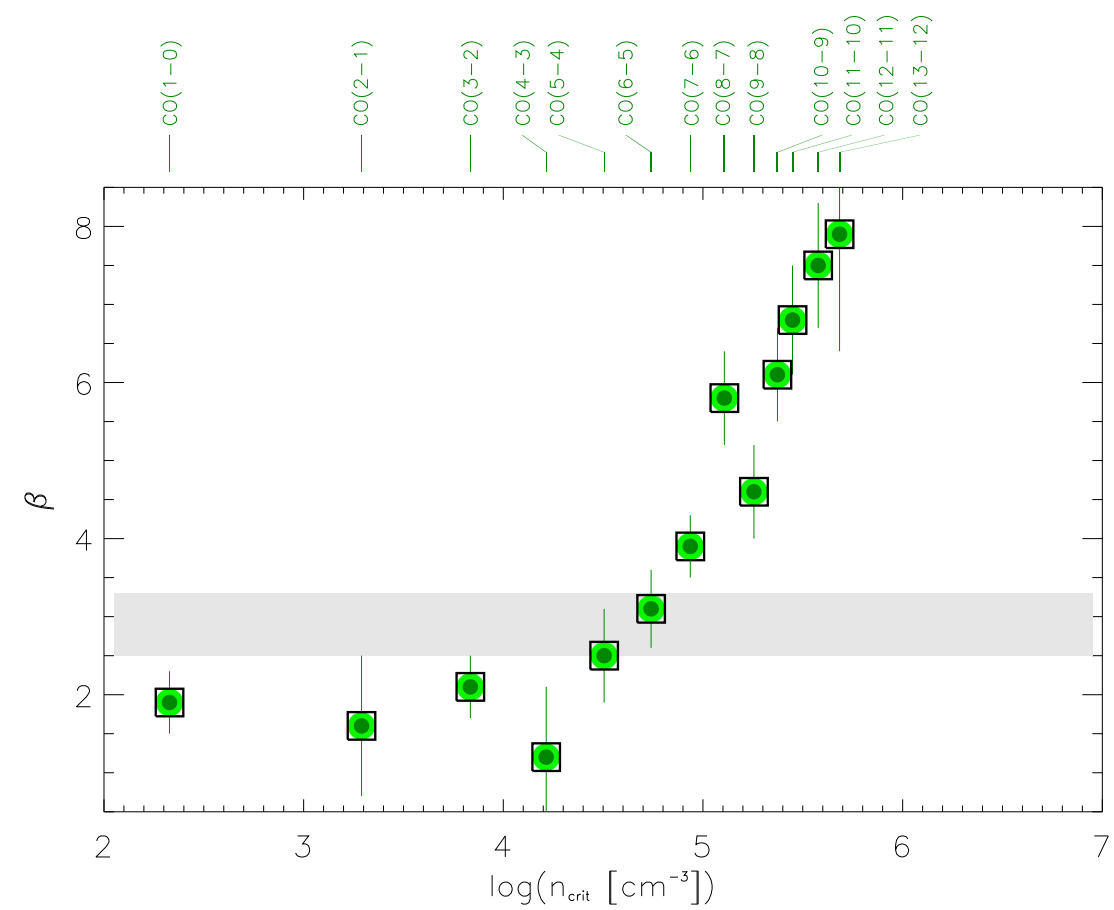

Figure 4. Normalizations $(\beta)$ of the FIR-CO relations presented in this paper (Figure 2 and Table 3). For $J=1-0$ to $5-4$ the normalizations are constant (within the errors), with values $(\beta \sim 1.2-2.5)$ close to that expected from Eddington limited star formation $(\sim 2.5-3.3$, shown as a gray shaded area), assuming a Rossland-mean opacity in the range $5-30 \mathrm{~cm}^{2} \mathrm{~g}^{-1}$ and a CO-to- $\mathrm{H}_{2}$ conversion factor of $0.8 \mathrm{~K} \mathrm{~km} \mathrm{~s}^{-1} \mathrm{pc}^{2}$ (see also Section 5.2). For $J=6-5$ and higher, the $\beta$-values increase with $J$ as the FIR-CO slopes become sublinear.

(A color version of this figure is available in the online journal.)

Table 4

Critical Densities $\left(n_{\text {crit }}\right)$ and Upper Level Energies $\left(E_{J} / k_{\mathrm{B}}\right)$ of the Rotational Ladder of CO, and Selected Transitions of HCN and CS, Assuming $\mathrm{H}_{2}$ is the Main Collision Partner

\begin{tabular}{lcr}
\hline \hline Transition & $\begin{array}{c}n_{\text {crit }} \\
\left(\mathrm{cm}^{-3}\right)\end{array}$ & $\begin{array}{c}E_{J} / k_{\mathrm{B}} \\
(\mathrm{K})\end{array}$ \\
\hline $\mathrm{CO}(1-0)$ & $3.09 \times 10^{2}$ & 5.53 \\
$\mathrm{CO}(2-1)$ & $2.73 \times 10^{3}$ & 16.60 \\
$\mathrm{CO}(3-2)$ & $9.51 \times 10^{3}$ & 33.19 \\
$\mathrm{CO}(4-3)$ & $2.29 \times 10^{4}$ & 55.32 \\
$\mathrm{CO}(5-4)$ & $4.48 \times 10^{4}$ & 82.97 \\
$\mathrm{CO}(6-5)$ & $7.70 \times 10^{4}$ & 116.16 \\
$\mathrm{CO}(7-6)$ & $1.21 \times 10^{5}$ & 154.87 \\
$\mathrm{CO}(8-7)$ & $1.78 \times 10^{5}$ & 199.11 \\
$\mathrm{CO}(9-8)$ & $2.50 \times 10^{5}$ & 248.88 \\
$\mathrm{CO}(10-9)$ & $3.41 \times 10^{5}$ & 304.16 \\
$\mathrm{CO}(11-10)$ & $4.63 \times 10^{5}$ & 364.97 \\
$\mathrm{CO}(12-11)$ & $6.00 \times 10^{5}$ & 431.29 \\
$\mathrm{CO}(13-12)$ & $7.55 \times 10^{5}$ & 503.13 \\
\hline $\mathrm{HCN}(1-0)$ & $1.07 \times 10^{5}$ & 4.25 \\
$\mathrm{HCN}(2-1)$ & $1.02 \times 10^{6}$ & 12.76 \\
$\mathrm{HCN}(3-2)$ & $3.52 \times 10^{6}$ & 25.52 \\
$\mathrm{HCN}(4-3)$ & $8.84 \times 10^{6}$ & 42.53 \\
\hline $\mathrm{CS}(1-0)$ & $6.77 \times 10^{3}$ & 2.35 \\
$\mathrm{CS}(2-1)$ & $6.50 \times 10^{4}$ & 7.05 \\
$\mathrm{CS}(3-2)$ & $2.40 \times 10^{5}$ & 14.11 \\
$\mathrm{CS}(5-4)$ & $1.34 \times 10^{6}$ & 35.27 \\
$\mathrm{CS}(6-5)$ & $2.36 \times 10^{6}$ & 49.37 \\
$\mathrm{CS}(7-6)$ & $3.76 \times 10^{6}$ & 65.83 \\
\hline & &
\end{tabular}

Notes. The $n_{\text {crit }}$-values are calculated for a kinetic temperature of $T_{\mathrm{k}}=40 \mathrm{~K}$, and an ortho- $\mathrm{H}_{2}:$ para- $\mathrm{H}_{2}$ ratio of 3 . are taken into account when fitting such relations, when in fact the uncertainties in both $L_{\mathrm{FIR}}$ and $L_{\mathrm{CO}}^{\prime}$ must be considered (see Mao et al. 2010 for a further discussion). Failing to do so can result in erroneous estimates of the slope.

For these reasons, we have refitted the data from a number of studies (see below) using the method described in Section 3, that is, with errors in both $L_{\mathrm{FIR}}$ and $L_{\mathrm{CO}}^{\prime}$ and including only sources with $L_{\text {FIR }} \gtrsim 10^{11} L_{\odot}$ in our analysis. Finally, not all studies use the FIR definition used here to infer $L_{\mathrm{FIR}}$, and other studies use the full $(8-1000 \mu \mathrm{m})$ luminosity. These differences can result in a different overall normalization (i.e., $\beta$ ), but are not expected to affect the determination of $\alpha$ (see Table 3 where there is little change in $\alpha$ when switching between $L_{\mathrm{FIR}[50-300 \mu \mathrm{m}]}$ and $\left.L_{\mathrm{IR}[8-1000 \mu \mathrm{m}]}\right)$.

From Figure 3 we note the overall good agreement between the FIR-CO slopes derived here and values from the literature. For $\mathrm{CO}(1-0)$, however, one set of measurements found superlinear slopes $\left(\alpha_{\mathrm{CO}_{1,0}} \sim 1.3-1.4\right.$; Juneau et al. 2009; Bayet et al. 2010), while most others favor a slope of unity (Gao \& Solomon 2004b; Mao et al. 2010; Ivison et al. 2011; this work). Note that our reanalysis of the Yao et al. (2003) and Baan et al. (2008) data revised their slopes from super-linear to linear: $\alpha_{\mathrm{CO}_{1.0}}=0.94 \pm 0.07$ and $1.08 \pm 0.06$, respectively (a similar result was found by Mao et al. 2010). Gao \& Solomon (2004b) finds a super-linear FIR-CO slope $(\alpha=1.3-1.4)$ from their entire sample (combining $L_{\mathrm{IR}} \sim 10^{10} L_{\odot}$ objects with LIRGs and ULIRGs), yet when including only the LIRGs and ULIRGs in the analysis, we obtain a linear slope $\left(\alpha_{\mathrm{CO}_{1,0}}=0.91 \pm 0.22\right)$. Refitting the data presented in Juneau et al. (2009) and Bayet et al. (2010) we reproduce their super-linear slopes.

For $\mathrm{CO}(2-1)$ our slope of unity is consistent within the errors with Bayet et al. (2010), who find a slightly super-linear slope based on 17 sources. Our reanalysis of the $\mathrm{CO}(2-1)$ data by Baan et al. (2008) yields a sublinear slope of $\alpha=0.82 \pm 0.10$. 
However, as pointed out by the authors, a nonnegligible fraction of the total $\mathrm{CO}(2-1)$ emission is likely to have been missed due to the smaller telescope beam at higher frequencies, thus biasing the FIR-CO relation to a shallower value of $\alpha$.

In the case of $\mathrm{CO}(3-2)$, the existing slope-determinations (Yao et al. 2003; Narayanan et al. 2005; Iono et al. 2009; Bayet et al. 2010; Mao et al. 2010), including our own, are in agreement and favor a value of unity within the errors. This includes a reanalysis of the Yao et al. (2003) data, which yielded $\alpha=1.00$ (see also Mao et al. 2010).

For $\mathrm{CO}(4-3)$ to $\mathrm{CO}(7-6)$ there is agreement within the errors between our results and the slopes found by Bayet et al. (2010), which were determined using observed and model-extrapolated CO luminosities of 7 low $-z$ and 10 high- $z$ sources. A departure from linear toward sublinear is also found by the latter study, albeit we find this turnover to occur at $J=6-5$, rather than at $J=4-3$ as deduced by Bayet et al. (2010). Here we must note, however, that the use of models to extrapolate to high$J$ CO luminosities is not safe, and artificial turnovers can be introduced because of the inability of such models-in the absence of appropriate line data - to reliably account for the existence of warmer and denser gas components. This further underscores the value of our observed $L_{\mathrm{FIR}}-L_{\mathrm{CO}}^{\prime}$ relations from $J=1-0$ to $J=13-12$ in safely determining such departures from linearity and/or in normalization before proceeding toward any interpretation based on ISM/SF physics.

\subsection{Super-linear Slopes: the Simplest Scenario}

The few super-linear slopes of FIR-CO luminosity relations for low- $J \mathrm{CO}$ lines that survive careful reanalysis (Juneau et al. 2009; Bayet et al. 2010) could be a byproduct of dense molecular gas being the direct SF fuel in all galaxies (with a constant SFE) and a $f_{\text {dense, } \mathrm{X}}=M_{\text {dense }} / M_{\mathrm{X}}$ (where $\mathrm{X}$ could be the total $\mathrm{H}_{2}$ gas mass traced by $\mathrm{CO} J=1-0, J=2-1$ lines), which varies within the galaxy sample with $d\left(f_{\text {dense }}\right) / d L_{\text {FIR }}>0$. Variations of this simple scenario have been suggested throughout the literature (Wong \& Blitz 2002; Gao \& Solomon 2004b), and unlike more sophisticated interpretations of such super-linear slopes offered by the two theoretical treatises available on this matter (Krumholz \& Thompson 2007; Narayanan et al. 2008), the only assumption here is that $d\left(f_{\text {dense }}\right) / d L_{\mathrm{FIR}}>0$. The latter is a well-documented fact as starbursts/mergers, which dominate the high- $L_{\mathrm{FIR}}$ end, are observed to have larger dense/ total gas-mass fractions than lower- $L_{\mathrm{FIR}}$ isolated disks (e.g., Gao \& Solomon 2004b; García-Burillo 2012).

Within this scheme the further the gas phase $\mathrm{X}$ is from the dense, star forming phase in terms of physical conditions and relevance to the star formation, the higher the $d\left(f_{\text {dense }}\right) / d L_{\mathrm{FIR}}$ value is and the deduced super-linear slope of the $L_{\mathrm{FIR}}-L_{\mathrm{X}}^{\prime}$ (or corresponding $\mathrm{S}-\mathrm{K}$ ) relation. On the other hand, for galaxy samples with a smaller range of IR-luminosities that have a nearly constant $f_{\text {dense, } X}$ - such as the (U)LIRG+DSFG sample considered in this paper (see Section 5.2) - a linear slope of an $L_{\mathrm{FIR}}-L_{\mathrm{X}}^{\prime}$ relation can still be recovered, for example, $\mathrm{CO}$ $J=1-0$, which traces all metal-rich molecular gas mass rather than only the dense SF one. We return to this point later in our discussion.

\section{CONFRONTING THEORETICAL MODELS}

\section{1. $\alpha$ versus $n_{\text {crit }}$}

As already mentioned in the Introduction, the S-K relation, and especially the important one involving the dense gas component in galaxies, is not easily accessible observationally. So one falls back to the much more observationally accessible proxy $L_{\mathrm{FIR}}-L_{\mathrm{X}}^{\prime}$ relations. Their index can then be linked to that of an assumed underlying $\mathrm{S}-\mathrm{K}$ relation in galaxies using two theoretical models (Krumholz \& Thompson 2007; Narayanan et al. 2008). Both models posit the same intrinsic $S-K$ relation of $\rho_{\mathrm{SFR}} \propto \rho_{\text {gas }}^{1.5}$ (or $\Sigma_{\mathrm{SFR}} \propto \Sigma_{\text {gas }}^{1.5}$ (for disks of near-constant scaleheight), justified under the assumption of a constant gas fraction transformed into stars per free fall time $\left(t_{\mathrm{ff}} \propto\left(G \rho_{\mathrm{gas}}\right)^{-1 / 2}\right)$. The same $\mathrm{S}-\mathrm{K}$ relation emerges if the SF timescale is instead set by the dynamical timescale of a marginally Toomre-stable galactic disk with SF converting a fixed fraction of gas into stars over such a timescale (Elmegreen 2002).

Both models give expected values of $\alpha$ versus $n_{\text {crit }}(\mathrm{X})$ for $L_{\mathrm{FIR}}-L_{\mathrm{X}}^{\prime}$ luminosity relations over a large range of line critical densities, but both models are applicable only for lines that require low temperatures to excite $E_{J} / k_{\mathrm{B}} \lesssim 30 \mathrm{~K}$ (for the Krumholz \& Thompson 2007 model this limit is $\sim 10 \mathrm{~K}$ ). The reasons behind this limitation are explicit assumptions about isothermal gas states at a set temperature (Krumholz \& Thompson 2007), or the tracking of such states over a small range of $T_{\mathrm{k}} \sim 10-30 \mathrm{~K}$ (Narayanan et al. 2008). The low- $J$ lines of heavy rotor molecular line (e.g. $\mathrm{HCN}$ and $\mathrm{HCO}^{+}$) data sets found in the literature and our low- $J \mathrm{CO}$ lines are certainly within the range of applicability of these models. The indices of the corresponding $L_{\mathrm{FIR}}-L_{\mathrm{X}}^{\prime}$ power law relations can thus be compared to these theoretical predictions.

Gao \& Solomon (2004a, 2004b) found a linear FIR-HCN correlation for the $J=1-0$ transition (see also Baan et al. 2008), which extends from local ULIRGs/LIRGs $\left(L_{\mathrm{IR}} \sim\right.$ $10^{11}$ to $\left.10^{12} L_{\odot}\right)$ to normal star forming galaxies $\left(L_{\mathrm{IR}} \sim\right.$ $10^{9}$ to $\left.10^{10} L_{\odot}\right)$, down to individual Galactic molecular clouds with $L_{\mathrm{IR}} \gtrsim 10^{4.5} L_{\odot}$ (Wu et al. 2005, 2010). A weakly superlinear FIR-HCN $(1-0)$ slope $(\alpha \simeq 1.2)$ was found by GraciáCarpio et al. (2008a) and García-Burillo (2012) over a combined sample of local normal galaxies and LIRG/ULIRGs. However, careful analysis by García-Burillo (2012) demonstrated that a bimodal fit (i.e., a different normalization parameter $\beta$ ) is better, with each galaxy sample well-fit by a linear relation. Finally, a weakly super-linear $L_{\mathrm{FIR}}-L_{\mathrm{HCN}_{1,0}}$ appears when high$z$ observations of the most IR-luminous starburst galaxies and QSOs are included in the locally established relation (Gao et al. 2007; Riechers et al. 2007). This could be bimodal instead, but with an otherwise linear $L_{\mathrm{FIR}}-L_{\mathrm{HCN}_{1,0}}$ relation (and with insufficient high- $L_{\text {FIR }}$ objects to decide the issue). A physical reason for such bimodalities is discussed in Section 5.2.

Extending HCN observations to include many more objects in the crucial $L_{\mathrm{IR}}>10^{12} L_{\odot}$ regime is necessary for deciding such issues. Even then one must eventually obtain the underlying SFR $-M_{\text {dense }}$ relation before arriving at secure conclusions about a varying $\mathrm{SFE}=\mathrm{SFR} / M_{\text {dense }}$ of the dense gas in (U)LIRGs. The latter is the crucial physical quantity underlying the normalization of such $L_{\mathrm{FIR}}-L_{\mathrm{HCN}}^{\prime}$ relations and, for example, a rising or bimodal $X_{\mathrm{HCN}}=M_{\mathrm{dense}} / L_{\mathrm{HCN}_{1,0}}$ factor toward high$L_{\mathrm{IR}}$ systems can easily erase purported SFE trends obtained by using single-line proxies of dense gas.

A sublinear FIR-HCN slope $(\sim 0.7-0.8)$ for the $J=3-2$ transition has been reported (Bussmann et al. 2008; Juneau et al. 2009), but is very likely biased low due to not having performed any beam correction (see Section 2) for some of their very nearby extended objects, where the $\mathrm{HCN}$ beam does not cover the entire IR emitting region. For these sources, the HCN measurements do not match the IR luminosities, and since they 
reside at the lower end of the $\mathrm{HCN}$ luminosity distribution, the net effect will be to bias the relation toward shallower values. For this reason we have chosen to ignore the sublinear FIR-HCN slopes from Bussmann et al. (2008) and Juneau et al. (2009). A recent survey of HCN $J=4-3$ and CS $J=7-6$ (Zhang et al. 2014), as well as CS $J=1-0,2-1,3-2$, and 5-4 (Z.-Y. Zhang et al., in preparation), toward nearby star forming galaxies $\left(L_{\mathrm{IR}} \sim 10^{9}-10^{12} L_{\odot}\right)$, where such effects have been adequately accounted for, establishes a slope $\alpha \sim 1$ for these transitions (see also Wu et al. 2010 and Wang et al. 2011). Many of these CS transitions have higher critical densities than $\mathrm{HCN}$, and $\mathrm{CS}$ is furthermore less prone to IR pumping effects than $\mathrm{HCN}$ (CS is pumped at $7.9 \mu \mathrm{m}$ compared to $14 \mu \mathrm{m}$ for $\mathrm{HCN}$ ). Pumping of HCN (and also HNC), however, typically only becomes important at dust temperatures $\gtrsim 50 \mathrm{~K}$ (Aalto et al. 2007), and would typically require even higher temperatures for CS. This is important because pumping could affect the $\mathrm{CS} / \mathrm{HCN}$ luminosities (especially at high- $J$ ), and thus result in linear IR-CS/HCN relations. In Figure 3 we summarize all the observationally determined $L_{\mathrm{IR}}-L_{\mathrm{HCN}}^{\prime}$ and $L_{\mathrm{IR}}-L_{\mathrm{CS}}^{\prime}$ slopes from the literature, along with those derived from our $\mathrm{CO}$ lines. Overall, the data suggest $\alpha \sim 1$ for our $L_{\mathrm{FIR}}-L_{\mathrm{CO}}^{\prime}$ relations from $J=1-0$ up to $J=5-4,6-5$, and for the HCN and CS lines. The latter cover a range of $n_{\text {crit }} \sim 10^{4}-10^{7} \mathrm{~cm}^{-3}$ (i.e., reaching up well into the high-density regime of the star forming gas phase).

From Figure 3 it becomes clear that most observations are incompatible with current model predictions (shown as the gray shaded area) both for the heavy rotor and the low- $J$ CO lines (where such models remain applicable). Super-linear slopes do appear for some CO $J=1-0$ data sets but then, unlike model predictions, the slopes remain linear for lines with much higher critical densities, including those of mid-J CO lines $J=3-2,4-3,5-4$ (the FIR-CO luminosity relation for $J=6-5$ is also compatible with a linear one).

In summary, we conclude that the global $L_{\mathrm{FIR}}-L_{\mathrm{X}}^{\prime}$ relations in (U)LIRGs and DSFGs, as parameterized by $\log L_{\mathrm{FIR}}=$ $\alpha \log L_{\mathrm{X}}^{\prime}+\beta$, are linear for heavy rotor lines, and our CO line data set up to $J=6-5$, at which point the relations become increasingly sublinear for higher $J$. Moreover, the normalization factor $\beta$ shows a similar behavior by being nearly constant $\beta \sim 2$ up to $J=5-4 / 6-5$, but then starting to increase systematically with increasing $J$, reaching $\beta \sim 8$ for $J=13-12$.

\subsection{More ISM Physics in $\beta$ Rather than in $\alpha$ ?}

The simplest scenario outlined in Section 4.2 seems to work both for the low-J CO and the HCN and CS lines with much higher critical densities. Given that our sample solely consists of (U)LIRGs (i.e., $L_{\mathrm{IR}[8-1000 \mu \mathrm{m}]} \geqslant 10^{11} L_{\odot}$ ), for which the dense gas fraction (i.e., $f_{\text {dense }}=L_{\mathrm{HCN}_{\text {low }-\mathrm{J}}}^{\prime} / L_{\mathrm{CO}_{\text {low }-\mathrm{J}}}^{\prime}$ ) is nearly constant (see discussion in Section 6), linear slopes are to be expected for low- $J$ FIR-CO relations. For other samples in the literature (e.g., Bayet et al. 2010) that reach lower IR luminosities $\left(\sim 10^{10} L_{\odot}\right)$ and thus span a wider range in $L_{\mathrm{IR}}$ (over which $f_{\text {dense }}$ changes appreciably) the super-linear slopes of their low- $J$ FIR-CO relations seen in Figure 3 are also expected. In this simple picture, neither the occasional super-linear nor the linear slope of the $L_{\mathrm{FIR}}-L_{\mathrm{CO}}^{\prime}$ low- $J$ relations carry any profound ISM physics other than that more dense gas mass corresponds to proportionally higher SFRs (as also suggested by Gao \& Solomon 2004b and Wu et al. 2005).
For dense gas tracer lines, this shows itself directly with $L_{\mathrm{IR}}-L_{\mathrm{X}}^{\prime}$ relations that always have linear slopes. Here there is actually more ISM physics to be found in exploring what sets the value of the normalization parameter $\beta$, rather than the slope of $L_{\mathrm{IR}}-L_{\mathrm{X}}^{\prime}$ relations.

The low- and high- $z$ (U)LIRGs studied here are highly dust-obscured galaxies, and the radiation pressure exerted by the strong absorption and scattering of FUV light by dust grains could be an important feedback mechanism, possibly setting the value of the normalization (and ultimately regulating the SF). The maximum attainable $L_{\mathrm{IR}} / M_{\text {dense }}$ ratio of a star forming region before radiation pressure halts higher accretion rates is ultimately set by the Eddington limit, giving, $L_{\mathrm{IR}} / M_{\text {dense }} \sim 500 L_{\odot} M_{\odot}{ }^{-1}$ (Scoville \& Polletta 2001). Andrews \& Thompson (2011) expressed the expected $L_{\mathrm{IR}}-L_{\mathrm{CO}}^{\prime}$ and $L_{\mathrm{IR}}-L_{\mathrm{HCN}}^{\prime}$ relations in the case of Eddington-limited SFRs, and found that for $\mathrm{CO}$ luminosity tracing only the active star forming gas the maximal possible luminosity is given by $L_{\mathrm{Edd}}=4 \pi G c \kappa^{-1} X_{\mathrm{CO}} L_{\mathrm{CO}}^{\prime}$, where $\kappa$ is the Rosseland-mean opacity, and $X_{\mathrm{CO}}$ is the $L_{\mathrm{CO}}^{\prime}$-to- $M_{\mathrm{H}_{2}}$ conversion factor. A similar expression holds for $\mathrm{HCN}$, albeit with different $\kappa$ and $X$ values (see Andrews \& Thompson 2011 for details). Although the exact normalization of this relation for each molecular line depends on poorly constrained quantities like $\kappa$ and $X$, the Eddington limit set by the strong FUV/optical radiation from embedded SF sites acting on the accreted dust and dense gas can naturally provide the normalization of the observed $L_{\mathrm{FIR}}-L_{\mathrm{CO}}^{\prime}$ and $L_{\mathrm{FIR}}-L_{\mathrm{HCN}}^{\prime}$ relations. In fact, adopting $\kappa=5-30 \mathrm{~cm}^{2} \mathrm{~g}^{-1}$ and $X_{\mathrm{CO}}=0.8 \mathrm{~K} \mathrm{~km} \mathrm{~s}^{-1} \mathrm{pc}^{2}$, which are perfectly reasonable values for (U)LIRGs (Thompson 2005; Solomon et al. 1997), we find $\beta=\log \left(4 \pi G c \kappa^{-1} X_{\mathrm{CO}}\right)=2.5-3.3$. We note that the high $\kappa$-value $\left(30 \mathrm{~cm}^{2} \mathrm{~g}^{-1}\right)$, which corresponds to the three-fold increase in the dust-to-gas mass ratio for the Rosseland-mean opacity (see Andrews \& Thompson 2011 for details) that might be expected in (U)LIRGs, yields $\beta=2.5$, which is close to the observed normalization values obtained $(\beta \simeq$ 2) for the low- $J$ CO lines in Section 3 (Table 3; see also Figure 4).

Given that the Eddington limit is ultimately set within individual SF sites embedded deep inside molecular clouds, it will operate on all galaxies, not just (U)LIRGs. For ordinary star forming spirals, the global $\beta$ normalization value of the $L_{\mathrm{FIR}}-L_{\mathrm{CO}}^{\prime}$ relations for low- $J$ CO lines will be lower than its (Eddington limit) set value by a factor approximately equal to the logarithm of its dense gas fraction, that is, $\log \left(f_{\text {dense }}\right)=\log \left(M_{\text {dense }} / M_{\text {tot }}\right)$. By the same token, the offset in the $\log \left(L_{\mathrm{FIR}}\right)-\log \left(L_{\mathrm{CO}}^{\prime}\right)$ plane between two populations with significantly different dense gas fractions (e.g., $f_{\text {dense, } 1}$ and $f_{\text {dense, } 2}$ ) can be shown to be $\Delta \beta \sim \log \left(f_{\text {dense, } 2} / f_{\text {dense }, 1}\right)$. Thus, an increasing $f_{\text {dense }}\left(L_{\mathrm{IR}}\right)$ function can cause the super-linear FIR- $\mathrm{CO}($ low- $J)$ relations seen in some galaxy samples (which in reality are a varying $\beta\left(L_{\mathrm{IR}}\right)$ rather than a superlinear $\alpha$ ).

Local (U)LIRGs and high-z DSFGs, on the other hand, form stars closer to the Eddington limit on a global scale (Andrews \& Thompson 2011). Thus, the linear FIR-CO (low- $J$ ) and FIR-HCN/CS relations observed for local (U)LIRGs and high- $z$ DSFGs is consistent with the notion that radiation pressure is an important physical mechanism that underlies the observed star formation laws in highly dust-obscured galaxies. In effect, the extreme merger/starbursts that dominate the (U)LIRGs and high- $z$ DSFG populations resemble dramatically scaled-up versions of dense gas cores hosting SF deep inside Giant Molecular Clouds (GMCs), with the balance between 
radiation pressure and self-gravity setting their equilibrium during the IR-luminous phase.

In this framework, the failure of the available theoretical models to account for the observed $L_{\mathrm{FIR}}-L_{\mathrm{CO}}^{\prime}$ relations of low- $J$ CO and heavy rotor molecular lines might be attributed to the role that radiation pressure feedback plays in ultimately determining such relations. This has not been taken into account in all current theoretical considerations that either seek to explain the S-K relation in galaxies (e.g., Elmegreen 2002), or use an $(\mathrm{S}-\mathrm{K})$ relation of $\rho_{\mathrm{SFR}} \propto\left(\rho_{\text {gas }}\right)^{1.5}$ to determine the emergent $L_{\mathrm{FIR}}-L_{\text {line }}^{\prime}$ relations for molecular gas (Krumholz \& Thompson 2007; Narayanan et al. 2008). These use self-gravity and the associated timescale of free-fall time, along with models on how the SF efficiency (gas mass fraction converted into stars per freefall time) varies per phase in turbulent gas as the main ingredients toward a complete understanding of SF, S-K relations, and the proxy $L_{\mathrm{FIR}}-L_{\mathrm{X}}^{\prime}$ relations. A nongravitational force like that exerted by radiation pressure on accreted gas and dust near SF sites can greatly modify such a picture by reducing or eliminating the dependence on the free fall time, especially for the high-density gas (which, presumably, is the one closest to active SF sites). Alternatively, it has been suggested that in lower luminosity systems the star formation may be regulated by feedback-driven turbulence (kinetic momentum feedback) rather than by radiation pressure (Ostriker \& Shetty 2011; Shetty \& Ostriker 2012; Kim et al. 2013). Assuming a continuum optical depth at FIR wavelengths $\left(\tau_{\mathrm{FIR}}\right)$ of the order of unity for our sample of starburst/merger (U)LIRGs and typical dust temperatures of $\sim 50 \mathrm{~K}$, we can make a rough estimate of the expected radiation pressure, namely $P_{\text {rad }} \sim \tau_{\mathrm{FIR}} \sigma T_{\mathrm{d}}^{4} / c \sim$ $1.2 \times 10^{-8} \mathrm{erg} \mathrm{cm}^{-3}$ (where $\sigma$ is Stefan-Boltzmann's constant and $c$ the speed of light). This is comparable to the turbulent pressure $P_{\text {turb }} \sim \rho \sigma_{\mathrm{v}}^{2} / 3 \sim 1.4 \times 10^{-8} \mathrm{erg} \mathrm{cm}^{-3}$, obtained assuming a turbulent velocity dispersion of $\sigma_{\mathrm{v}} \sim 5 \mathrm{~km} \mathrm{~s}^{-1}$ and an average gas mass density of $\rho \sim 2 \mu n_{\mathrm{H}_{2}}$ corresponding to $n_{\mathrm{H}_{2}} \sim 10^{5} \mathrm{~cm}^{-3}$. Both of these greatly exceed the expected thermal pressure $-P_{\text {th }} \sim n_{\mathrm{H}_{2}} k_{\mathrm{B}} T_{\mathrm{k}} \sim 1.4 \times 10^{-9} \mathrm{erg} \mathrm{cm}^{-3}$ (for $n_{\mathrm{H}_{2}} \sim 10^{5} \mathrm{~cm}^{-3}$ and $T_{\mathrm{k}} \sim 100 \mathrm{~K}$ ) - thus highlighting the point made previously that a complete physical model of the S-K relations has to incorporate the effects of radiation pressure and/or turbulence.

\section{THE $\alpha$ AND $\beta$ TURNOVERS FOR HIGH-J CO LINES}

Higher than $J=6-5$ neither the slope, $\alpha$, nor the normalization, $\beta$, of the $L_{\mathrm{FIR}}-L_{\mathrm{CO}}^{\prime}$ relations remain constant, but $\alpha$ decreases while $\beta$ increases toward higher $J$ levels. This can be understood using a simple argument first put forth (in a slightly different form than here) by Wong \& Blitz (2002). Consider that $\alpha_{\mathrm{CO}_{J, J-1}}=d \log L_{\mathrm{FIR}} / d \log L_{\mathrm{CO}_{J, J-1}}^{\prime}$ can be expressed as:

$$
\begin{aligned}
\alpha_{\mathrm{CO}_{J, J-1}} & =\frac{d \log L_{\mathrm{FIR}}}{d \log L_{\mathrm{HCN}_{1,0}}^{\prime}} \times \frac{d \log L_{\mathrm{HCN}_{1,0}}^{\prime}}{d \log L_{\mathrm{CO}_{J, J-1}}^{\prime}} \\
& =\alpha_{\mathrm{HCN}_{1,0}}\left(1+\frac{d \log l_{\mathrm{dense}_{J, J-1}}}{d \log L_{\mathrm{CO}_{J, J-1}}^{\prime}}\right),
\end{aligned}
$$

where $\alpha_{\mathrm{HCN}_{1,0}}$ is the slope of the FIR-HCN $J=1-0$ relation, which is near unity, as mentioned previously. The last term, $l_{\text {dense }_{J, J-1}}=L_{\mathrm{HCN}_{1,0}}^{\prime} / L_{\mathrm{CO}_{J, J-1}}^{\prime}$, is a convenient parameterization of deviations in $\alpha_{\mathrm{CO}_{J, J-1}}$ from unity, and depends on both the

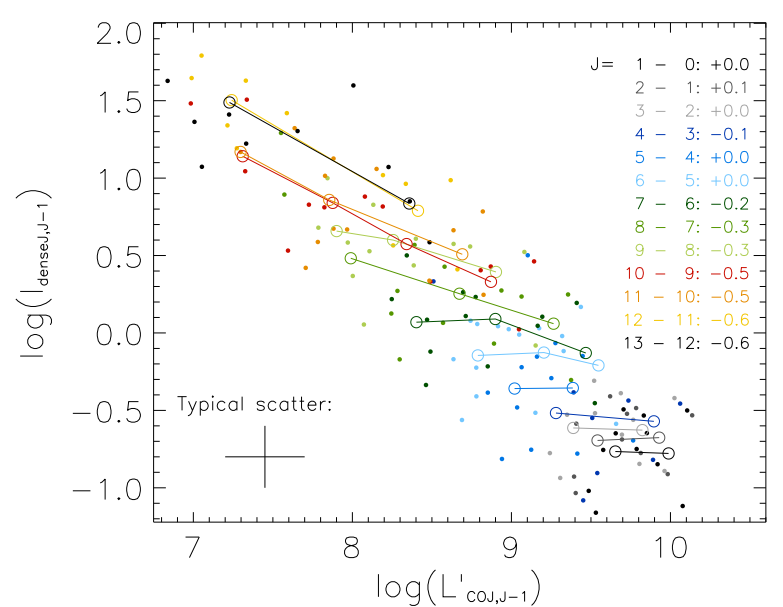

Figure 5. $\log l_{\text {dense }_{J, J-1}}$ vs. $\log L_{\mathrm{CO}_{J, J-1}}^{\prime}$ for our local (U)LIRG sample defined in Section 2 (solid symbols), where $l_{\text {dense }_{J, J-1}}=L_{\mathrm{HCN}_{1,0}}^{\prime} / L_{\mathrm{CO}_{J, J-1}}^{\prime}$ (see Section 6). The different $\mathrm{CO}$ transitions are color-coded (see inset). To highlight the trends, we show the average $\log l_{\text {dense }_{J, J-1}}$ values within suitable bins of $L_{\mathrm{CO}_{J, J-1}}^{\prime}$ (shown as open circles and connected with solid lines). Linear fits to these averages yield the slopes given in the inset. For $\mathrm{CO}(1-0)$ to $\mathrm{CO}(6-5)$, we find $d \log l_{\text {dense }_{J, J-1}} / d \log L_{\mathrm{CO}_{J, J-1}}^{\prime} \simeq 0$, which when inserted in Equation (3) yields $\alpha_{\mathrm{CO}_{J J-1}} \simeq 1$, in agreement with our findings. For higher $\mathrm{CO}$ transitions, we have $d \log l_{\text {dense }_{J, J-1}} / d \log L_{\mathrm{CO}_{J, J-1}}^{\prime}<0$, which results in the sublinear $\alpha_{\mathrm{CO}_{J, J-1}}$-values that match our directly determined FIR-CO slopes.

(A color version of this figure is available in the online journal.)

dense gas content (as traced by $\mathrm{HCN}$ ) and the global $\mathrm{CO}$ line excitation.

For CO $J=1-0, l_{\text {dense }_{1,0}}$ is a linear proxy of the dense gas mass fraction. This is simply due to the linearity of the $L_{\mathrm{HCN}_{1,0}}^{\prime}-L_{\mathrm{CO}_{1,0}}^{\prime}$ relation (a fit to the $L_{\mathrm{IR}[8-1000 \mu \mathrm{m}]}>10^{11} L_{\odot}$ sources in the Gao \& Solomon 2004b sample yield $\log L_{\mathrm{HCN}_{1,0}}^{\prime} \simeq$ $0.9 \log L_{\mathrm{CO}_{1,0}}^{\prime}-0.2$ ), and the fact that $\mathrm{CO} J=1-0$ provides a good linear measure of $M_{\text {tot }}\left(\mathrm{H}_{2}\right)$. The same applies for the $J=2-1$ line. For the higher $J$ CO lines, $n_{\text {crit }}$ becomes similar to that of HCN $J=1-0$ (or only slightly surpasses it) while their $E_{J} / k_{\mathrm{B}}(\sim 115-500 \mathrm{~K})$ significantly exceed that of $\mathrm{HCN}$ $J=1-0(\sim 4.3 \mathrm{~K})$. The high- $J$ CO lines are significantly excited (see Section 6.1) and, following the argument first made by Bradford et al. (2003), this is unlikely to be a pure density effect, as it would imply too large $\mathrm{CO} J=1-0$ and $2-1$ optical depths and, in turn, ${ }^{12} \mathrm{CO} /{ }^{13} \mathrm{CO}$ line ratios well below the typical values $(\sim 10-30)$ observed for local (U)LIRGs (e.g., Casoli et al. 1992; Aalto et al. 1995). Instead, we argue that the high- $J \mathrm{CO}$ lines are produced by a dense and warm $\left(T_{\mathrm{k}} \gtrsim 100 \mathrm{~K}\right)$ phase. The $l_{\text {dense }_{J, J-1}}$ then becomes a measure of the $R_{\mathrm{d}, \mathrm{d}-\mathrm{w}}=M_{\text {dense }}\left(\mathrm{H}_{2}\right) / M_{\text {dense-warm }}\left(\mathrm{H}_{2}\right) \geqslant 1$ modulo gas excitation differences between the dense (d) and the dense and warm $(\mathrm{d}-\mathrm{w})$ molecular gas reservoirs. The derivative inside the parenthesis in Equation (3) will be nearly zero for both low- and high- $J$ CO lines as long as (1) the dense gas mass fraction remains nearly constant within our galaxy sample (i.e., the sample is homogeneous in terms of $f_{\text {dense }}$ and its proxies $l_{\text {dense }_{1,0}}, l_{\text {dense }_{2,1}}$ ), and (2) the $R_{\mathrm{d}, \mathrm{d}-\mathrm{w}}$ ratio also remains constant. The latter means that the relative excitation conditions and mass between the dense gas component (d) and its subcomponent of dense and warm gas (d-w) remain invariant across the sample. The trend of $L_{\mathrm{FIR}}-L_{\mathrm{CO}}^{\prime}$ relations above $J=6-5$ toward increasing sublinearity for higher $J$ levels is due to a decrease of $l_{\text {dense }_{J, J-1}}$ with increasing high- $J$ CO luminosity, thus resulting in $\alpha_{\mathrm{CO}_{J, J-1}}<1$. This behavior is indeed obvious in Figure 5, 
which shows $l_{\text {dense }_{J, J-1}}$ as a function of $L_{\mathrm{CO}_{J J-1}}^{\prime}$ for a subset of our local (U)LIRG sample with HCN (1-0) detections from Gao \& Solomon (2004a). Note that we have not included DSFGs in this plot because most detections of $\mathrm{HCN}$ at high redshifts are of QSOs and AGN dominated DSFGs. In conjunction with Equation (3), Figure 5 can account for our established FIR-CO slopes in Figure 3 and Table 3.

A decreasing $l_{\text {dense }_{J J-1}}$ with increasing high- $J$ CO luminosity (yielding a negative derivative inside the parenthesis in Equation (3)) indicates increasing mass and/or excitation conditions of the warm and dense (d-w) gas component, relative to the dense gas reservoir (d) that presumably contains it. This is possible if galaxies with increasingly larger high- $J$ CO line luminosities (and thus also SFRs) increasingly have a warm and dense gas component no longer tied to their SF via the average FUV/optical radiation field. Such examples have been found for individual starbursts or star forming galactic nuclei (Bradford et al. 2003; Ward et al. 2003; Hailey-Dunsheath et al. 2008; Panuzzo et al. 2010; van der Werf et al. 2010; Rangwala et al. 2011; Meijerink et al. 2013; Rosenberg et al. 2014a), while the presence of large masses of such a molecular gas component was recently suggested as a general feature of the ISM in extreme merger/starbursts (Papadopoulos et al. 2012). High cosmic ray (CR) energy densities and/or the dissipation of galaxy-wide shocks due to strong supersonic turbulence can maintain $T_{\mathrm{k}} \gtrsim 100 \mathrm{~K}$ for large amounts of high-density gas, even in the absence of FUV radiation fields (e.g., Ao et al. 2013). Appreciable fractions of dense gas mass per GMC above such temperatures demand different heating mechanisms that can strongly heat the gas without readily dissociating $\mathrm{CO}$ as FUV radiation does, and without being attenuated by dust (i.e., $\mathrm{CR}$ - and turbulent heating). The onset of increasing normalization factors, $\beta$, of the $L_{\mathrm{FIR}}-L_{\mathrm{CO}}^{\prime}$ relations above $J=6-5$ is then simply another result of the weakening link between the FUVpowered $L_{\mathrm{FIR}}$ and the thermal state of dense gas for systems with high SFRs (and high- $J$ CO line luminosities). The rapid rise of $\beta$ with $J$-level is expected if CO lines at increasingly higher- $J$ levels probe ever higher gas thermal states with smaller mass per IR luminosity.

The above picture retains the simple explanation for the observed $\alpha \simeq 1$ for the $L_{\mathrm{FIR}}-L_{\mathrm{CO}}^{\prime}$ relations from $J=1-0$ to $J=5-4$, and for those found for several heavy rotor molecular lines, as long as all these relations refer to a near $f_{\text {dense }^{-}}$ homogeneous galaxy sample, with SF powering both the dust continuum and the molecular line luminosity via FUV radiation. Highly super-linear slopes can only occur for galaxy samples with significantly different dense gas fractions, or different star formation relation normalizations (e.g., Gao \& Solomon 2004b). Finally, in this overall scheme, and for good (i.e., linear) dense SF gas tracers, such as $\mathrm{HCN}$ and $\mathrm{CS}$, it is rather hard to envisage how sublinear slopes can come about (Juneau et al. 2009), because even the high- $J$ transitions of these molecules will trace the dense, cold star forming gas. Thus, the second term in Equation (3) will remain close to zero, leaving the FIR-HCN (or FIR-CS) relation linear. In fact, linear slopes are observed for transitions as high as CS $J=7-6$ (Zhang et al. 2014).

\subsection{The CO SLEDs and the Thermal State of High Density Gas}

A more direct indication of significant amounts of warm and dense gas in our (U)LIRG-dominated sample, and to what extent
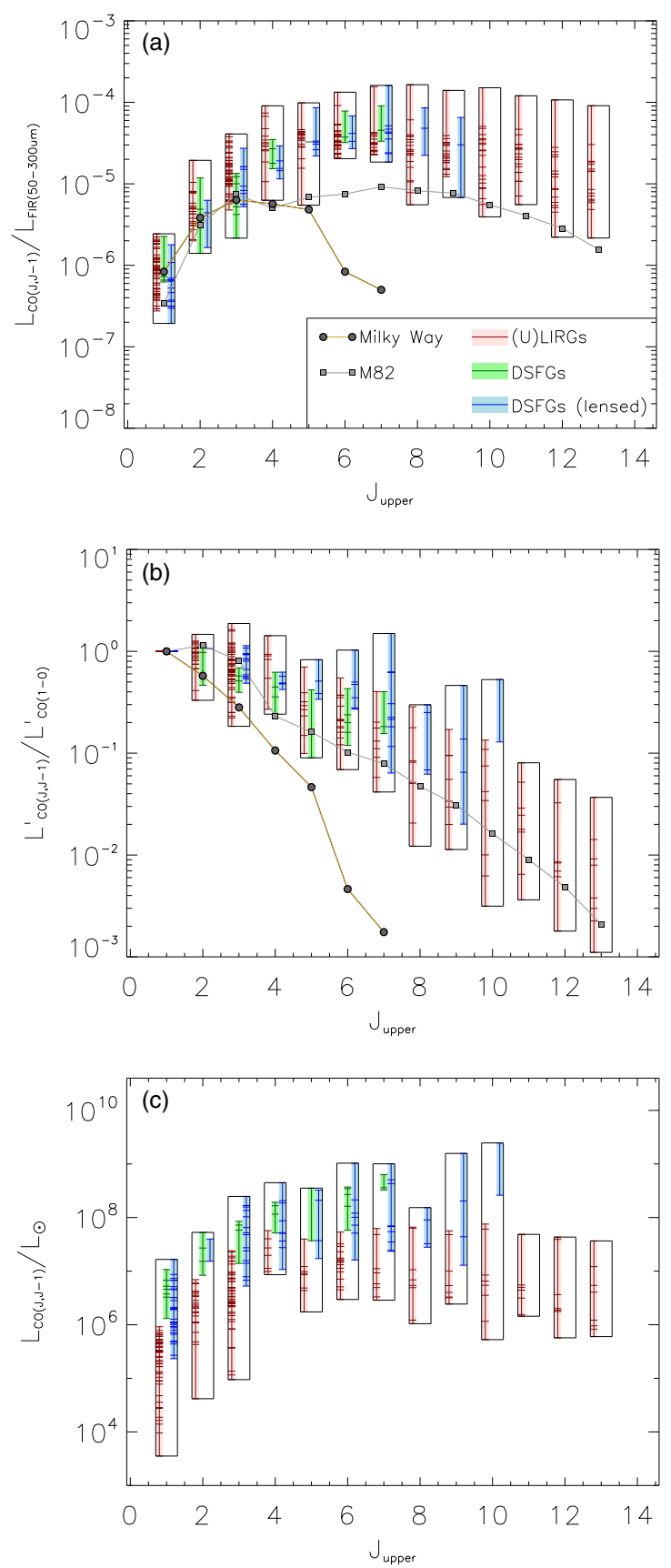

Figure 6. CO spectral energy distributions for the local (U)LIRG+HerCULES sample (red), the non-lensed (green), and strongly lensed (blue) high- $z$ DSFGs. The CO SLEDs are given as the CO line luminosities, in $L_{\odot}$ units normalized by the FIR luminosity (top), as brightness temperature ratios, that is, $L_{\mathrm{CO}_{\mathrm{J}, \mathrm{J}-1}^{\prime}} / L_{\mathrm{CO}_{1,0}}^{\prime}$ (middle), and simply as $L_{\mathrm{CO}_{\mathrm{J}, \mathrm{J}-1}}$ (in $L_{\odot}$ units) versus $J$ (bottom). The filled bars indicate the full range of $L_{\mathrm{CO}_{J, J-1}} / L_{\mathrm{FIR}}-$ and $L_{\mathrm{CO}_{\mathrm{J} J \mathrm{~J}-1}}^{\prime} / L_{\mathrm{CO}_{1,0}}^{\prime}$ values in the two panels, respectively, whereas the tick marks indicate the values of individual sources. For comparison we also show the $\mathrm{CO}$ SLEDs for the inner Galaxy (up to $J=7-6$ ) as measured by FIRAS/COBE (Fixsen et al. 1999), normalized by $L_{\mathrm{FIR}}=(1.8 \pm 0.6) \times 10^{10} L_{\odot}$ (also measured by FIRAS; Wright et al. 1991) and for the proto-typical nearby starburst galaxy M 82 (Panuzzo et al. 2010).

(A color version of this figure is available in the online journal.)

its thermal state is likely to be maintained by the SFR-powered average FUV radiation fields, is provided by the CO SLEDs. In Figure 6 we show the FIR- and $\mathrm{CO}(1-0)$-normalized $\mathrm{CO}$ SLEDs (top and middle panels, respectively), as well as the raw 
CO luminosities (bottom panel). The first version allows for an assessment of the CO SLEDs for the full samples (not all of our sources have $\mathrm{CO} J=1-0$ measurements), and shows the cooling power of the $\mathrm{CO}$ lines with respect to the continuum. The CO $J=1-0$ normalized representation of the CO SLEDs makes for a direct comparison with observed $\mathrm{CO}$ line ratios in the literature, and is furthermore what is usually used to constrain the excitation conditions of the gas.

A detailed analysis of the CO SLEDs, in conjunction with the multi- $J \mathrm{HCN}, \mathrm{CS}$, and $\mathrm{HCO}^{+}$line data setsavailable for many of the (U)LIRGs in Figure 6, is needed for a full understanding of the heating and cooling mechanisms of the molecular gas and for quantifying the relative mass-fractions of the gas phases. Nevertheless, the marked contrast between their CO SLEDs and that of the Milky Way disk (where most of bulk of the molecular gas is warmed by photoelectric heating induced by the ambient FUV radiation field), already indicates the presence of a different heating source. While intense X-ray radiation fields $(1-5 \mathrm{keV})$ generated by AGN can penetrate and heat gas up to $\gtrsim 100 \mathrm{~K}$ at column densities of $10^{22}-10^{24} \mathrm{~cm}^{-2}$ (and without dissociating all the $\mathrm{CO}$ ), it is unlikely to be the case here because great care has been taken in removing AGNs from our sample. The integrated power emitted in the CO $J=7-6$ to $J=13-12$ transitions for all the (U)LIRGs in our sample constitutes the bulk (about $60 \%$ ) of the total energy output of all the $\mathrm{CO}$ lines. Exploring the effects of different CR and mechanical heating rates on the thermal structure of clouds, Meijerink et al. (2011) found that even for extreme CR fluxes $\left(\sim 10^{2}-10^{4} \times\right.$ the Milky Way value) it is difficult to maintain temperatures $\gtrsim 100 \mathrm{~K}$, and the effect on the high- $J$ CO lines appears to be minor. Mechanical heating, such as supernova driven turbulence and shocks, however, was found to heat the gas more efficiently, and we favor this as the most likely explanation for hot gas and the boosted high- $J$ CO lines observed in our (U)LIRGs.

Highly excited CO SLEDs have been found for the merger/ starburst NGC 6240, where a recent analysis found FUV photons (and the resulting photoelectric heating) to be inadequate as the main heating source for the high temperatures of its dense gas (Meijerink et al. 2013; Papadopoulos et al., 2014), and for Mrk 231 where X-rays from the AGN are thought to heat the dense molecular gas reservoir (van der Werf et al. 2010; although Mrk 231 has recently been shown to be much less X-ray luminous than previously thought; see Teng et al. 2014). While such CO SLEDs, and the need for alternative heating mechanisms than FUV-photons to explain them, might be linked to the unusually high $\mathrm{CO}$ line-to-continuum ratios of both of these two sources, a similar conclusion was reached for M 82 and NGC 253 based on analyses of their full CO SLEDs (Panuzzo et al. 2010; Rosenberg et al. 2014a). Our work is the first to demonstrate such highly excited high$J$ CO SLEDs as a near generic characteristic of merger/ starbursts (the galaxies that dominate the sample shown in Figure 6).

\subsection{Some Possible Caveats}

As mentioned in Section 2, incorrect FIR-CO relations may be inferred if the FIR and CO measurements cover different regions within galaxies. This can be a serious problem for local extended sources where single-dish $\mathrm{CO}$ beams can be smaller than the extent of the IR emission (see discussion in Zhang et al. 2014). We are confident that this is not an issue for our HerCULES sample, where all SPIRE-FTS CO line fluxes were scaled to a common $42^{\prime \prime}$ angular resolution, which is beyond the extent of the IR emission in these sources (as traced by LABOCA $870 \mu \mathrm{m}$ maps). However, if this was not the case, and the SPIRE-FTS measurements did not capture all the $\mathrm{CO}$ emission, it would imply that the derived FIR-CO slopes are biased high (since the CO luminosity will be underestimated relative to the total FIR luminosity, see Figure 2). In short, the sublinear FIR-CO slopes at high- $J$ transition found here are robust against the (unlikely) possibility that some (small) fraction of the $\mathrm{CO}$ emission is unaccounted for.

The effects that the presence of strong AGNs would have on the SF relations are two-sided. On the one hand, it could lead to an overestimate of the IR luminosity attributed to star formation, thus biasing the FIR-CO slopes high. On the other hand, AGN-dominated environments, where penetrating X-rays may be dominating the gas heating, tend to have boosted high$J$ CO lines compared to star forming regions (e.g., Meijerink et al. 2007). If the AGN was deeply buried it would not be easily detectable in X-rays and would be optically thick in the IR, possibly down to mm wavelengths. The effect could be what is observed here-a change of slope in the correlation and the high-J CO lines reflecting a hot, deeply embedded AGN. As mentioned in Section 2, we used $L_{\text {FIR }[50-300 \mu \mathrm{m}]}$ instead of $L_{\mathrm{IR}[8-1000 \mu \mathrm{m}]}$ in the FIR-CO relations in order to minimize the effects of AGN. More importantly, we only included local (U)LIRGs for which the bolometric AGN contribution was deemed to be $<20 \%$, as estimated from several MIR diagnostics (Section 2). In the case of the high- $z$ sample, systems that were obviously AGN-dominated were immediately discarded (Section 2). Furthermore, we note that deep X-ray observations and MIR spectroscopy of millimeter and submillimeter selected DSFGs (which show no obvious signs of harboring an AGN) have shown that any AGN that might be present typically contribute $\lesssim 20 \%$ to the total IR luminosity (Alexander et al. 2005; Mendez-Delmestre et al. 2007). Thus, we feel confident that neither the FIR nor the CO luminosities are biased high due to AGNs, and therefore our findings are not systematically affected by AGNs.

Galaxies that are gravitationally lensed are prone to differential magnification, an effect in which regions within a galaxy are magnified by different amounts due to variations in their location within the galaxy, and/or spatial extent (Blain 1999). This can significantly skew the observed relative contributions from hot versus cold dust to the IR luminosity, as well as lowversus high-JCO line luminosity ratios (Serjeant 2012). Furthermore, a flux-limited sample of strongly lensed sources will tend to preferentially select compact sources (Hezaveh et al. 2012), which may be more likely to have extreme $\mathrm{CO}$ excitation conditions. From Figure 6(a), however, there is nothing to suggest that the lensed DSFGs have markedly different $L_{\mathrm{CO}_{J, J-1}^{\prime}}^{\prime} / L_{\mathrm{FIR}}$ values than the nonlensed and local (U)LIRGs. In Figure 6(b), however, we do see a few lensed DSFGs that have markedly higher $L_{\mathrm{CO}_{J, J-1}}^{\prime} / L_{\mathrm{CO}_{1,0}}^{\prime}$ ratios at high- $J$ than the other samples. This is exactly what we would expect to see if these sources were differentially lensed (and the high- $J$ lines tracing more compact regions than the $J=1-0$ line), or if the lensing preferentially selects compact sources (which would tend to have more extreme excitation conditions). A strong argument against our analysis being affected by differential magnification effects is the fact that when fitting the FIR-CO relations without the lensed DSFGs we obtain slopes nearly identical to the ones given in Table 3, and fully consistent within the errors. Thus, we conclude that our findings are unlikely to be affected in any significant way by differential magnification effects. 


\section{SUMMARY}

Utilizing Herschel/SPIRE-FTS observations of a statistically significant sample of 23 local (U)LIRGs, simultaneously covering the $\mathrm{CO} J=5-4$ to $J=13-12$ lines in one single spectrum, and combining these with $\mathrm{CO} J=1-0,2-1,3-2,4-3$, and 6-5 data from our comprehensive ground-based $\mathrm{CO}$ survey of the same sample, as well for an additional 44 local (U)LIRGs, we have presented FIR-CO luminosity relations for the full CO rotational ladder from $J=1-0$ to $J=13-12$. Included in our analysis is a carefully groomed sample of 35 high- $z$ lensed and non-lensed DSFGs (spanning the redshift range $z \sim 1-6$ ) with robust FIR and CO luminosity measurements. Due to high redshifts, many of these sources have been observed in the midto high- $J \mathrm{CO}$ lines from the ground, thus allowing us to extend the mid- to high- $J$ FIR-CO relations to the highest redshifts.

For this data set of low- and high- $z$ merger/starburst dominated galaxies, we find linear $(\alpha \simeq 1)$ FIR-CO relations for CO $J=1-0$ up to $J=5-4$, with nearly constant normalization $(\beta \sim 2)$. In light of the linear star formation relations found for HCN and CS (e.g., Gao \& Solomon 2004b; Zhang et al. 2014, both of which are bona fide tracers of dense star forming gas, we have shown that our results are to be expected, provided the dense gas-mass fraction does not change significantly within the sample. Our findings are also qualitatively consistent with models in which the star formation in (U)LIRGs in the central $\sim 1 \mathrm{kpc}$ starburst region) is regulated by radiation pressure as these predict linear $L_{\mathrm{FIR}}-L_{\mathrm{mol}}$ slopes for any molecule/ transition that traces star forming gas in a homogeneous sample (i.e., constant normalization; Andrews \& Thompson 2011).

For CO $J=6-5$ and up to $J=13-12$, we find increasingly sublinear slopes and higher normalization constants, which we argue are due to these lines effectively being detached from the star formation, because they trace gas that is dense $\left(\gtrsim 10^{4} \mathrm{~cm}^{-3}\right)$ but also radically warmer ( $\gtrsim 100 \mathrm{~K})$ than what is typical for star forming gas. This dense and warm ISM component is reflected in the global CO SLEDs of the (U)LIRGs, and of the high- $z$ DSFGs, which remain highly excited from $J=6-5$ up to $J=13-12$. This suggests that star formation powered by FUV radiation fields is unlikely to be responsible for maintaining the gas temperature, and alternative heating sources are required instead. Mechanical heating via shocks/turbulence seems to be the most plausible alternative, given its effectiveness (compared to CRs) at driving the temperatures in clouds to the required levels $(\sim 100 \mathrm{~K})$.

Finally, we note that our derived FIR-CO relations are sufficiently tight, especially for the high- $J$ lines, that they can predict the expected CO line brightness of high- $z$ DSFGs, which in turn might be useful for planned observations with ALMA.

The authors gratefully acknowledge financial support under the "DeMoGas" project. The project DeMoGas is implemented under the "ARISTEIA" Action of the "Operational Programme Education and Lifelong Learning." The project is co-funded by the European Social Fund (ESF) and National Resources. T.R.G. acknowledges support from an STFC Advanced Fellowship. T.R.G. was also supported by Chinese Academy of Sciences Fellowship for Young International Scientists (grant no. 2012y1ja0006). Z.Y.Z. acknowledges support from the European Research Council (ERC) in the form of Advanced Grant,COSMICISM. We are indebted to P. P. Papadopoulos for extensive discussions and comments on the paper (He ho'okele wa'a no ka la 'ino). Basic research in infrared astron- omy at the Naval Research Laboratory is funded by the Office of Naval Research. J.F. also acknowledges support from the NHSC/JPL. The research presented here has made use of the NASA/IPAC Extragalactic Database (NED), which is operated by the Jet Propulsion Laboratory, California Institute of Technology, under contract with the National Aeronautics and Space Administration. Finally, we would like to thank the anonymous referee for a useful and constructive referee report that helped improve the paper.

\section{REFERENCES}

Aalto, S., Booth, R. S., Black, J. H., \& Johansson, L. E. B. 1995, A\&A, 300,369

Aalto, S., Spaans, M., Wiedner, M. C., \& Hüttemeister, S. 2007, A\&A, 464, 193 Akritas, M. G., \& Siebert, J. 1996, MNRAS, 278, 919

Alaghband-Zadeh, S., Chapman, S. C., Swinbank, A. M., et al. 2013, MNRAS, 435,1493

Alexander, D. M., Bauer, F. E., Chapman, S. C., et al. 2005, ApJ, 632, 736

Andrews, B. H., \& Thompson, T. A. 2011, ApJ, 727, 97

Ao, Y., Henkel, C., Menten, K. M., et al. 2013, A\&A, 550, 135

Aravena, M., Murphy, E. J., Aguirre, J. E., et al. 2013, MNRAS, 433, 498

Armus, L., Mazzarella, J. M., Evancs, A. S., et al. 2009, PASP, 121, 559

Baan, W. A., Henkel, C., Loenen, A. F., Baudry, A., \& Wiklind, T. 2008, A\&A, 477,747

Bayet, E., Gerin, M., Phillips, T. G., \& Contoursi, A. 2009, MNRAS, 399, 264 Bigiel, F., Leroy, A., Walter, F., et al. 2008, AJ, 136, 2846

Blain, A. W. 1999, MNRAS, 304, 669

Blain, A. W., \& Longair, M. S. 1993, MNRAS, 264, 509

Bothwell, M. S., Aquirre, M. S., Chapman, S. C., et al. 2013a, ApJ, 779, 67

Bothwell, M. S., Smail, I., Chapman, S. C., et al. 2013b, MNRAS, 429, 3047

Bradford, C. M., Nikola, T., Stacey, G. J., et al. 2003, ApJ, 586, 891

Burgarella, D., Baut, V., \& Iglesias-Páramo, J. 2005, MNRAS, 360, 1413

Bussmann, R. S., Gurwell, M. A., Fu, H., et al. 2012, ApJ, 756, 134

Bussmann, R. S., Narayanan, D., Shirley, Y. L., et al. 2008, ApJL, 681, L73

Bussmann, R. S., Pérez-Fournon, I., Amber, S., et al. 2013, ApJ, 779, 25

Calzetti, D., Kinney, A. L., \& Storchi-Bergmann, T. 1994, ApJ, 429, 582

Carilli, C. L., \& Walter, F. 2013, ARA\&A, 51, 105

Chary, R., \& Elbaz, D. 2001, ApJ, 556, 562

Casoli, F., Dupraz, C., \& Combes, F. 1992, A\&A, 264, 55

Conley, A., Cooray, A., Vieira, J. D., et al. 2011, ApJL, 732, L35

Cox, P., Krips, M., Neri, R., et al. 2011, ApJ, 740, 63

Daddi, E., Dannerbauer, H., Krips, M., et al. 2009, ApJL, 695, L176

Dale, D. A., \& Helou, G. 2002, ApJ, 576, 159

Danielson, A. L. R., Swinbank, A. M., Smail, I., et al. 2011, MNRAS, 410, 1687

Dopita, M. A., \& Ryder, S. D. 1994, ApJ, 430, 163

Downes, D., \& Solomon, P. M. 2003, ApJ, 582, 37

Elmegreen, B. G. 2002, in ASP Conf. Ser. 285, Modes of Star Formation and the Origin of Field Populations, ed. E. K. Grebel \& W. Brandner (San Francisco, CA: ASP), 425

Engel, H., Tacconi, L. J., Davies, R. I., et al. 2010, ApJ, 724, 233

Fixsen, D. J., Bennett, C. L., \& Mather, J. C. 1999, ApJ, 526, 207

Frayer, D. T., Harris, A. I., Baker, A. J., et al. 2011, ApJL, 726, L22

Frayer, D. T., Ivison, R. J., Scoville, N. Z., et al. 1999, ApJL, 514, 13

Frayer, D. T., Koda, J., Pope, A., et al. 2008, ApJL, 680, L21

Freundlich, J., Combes, F., Tacconi, L. J., et al. 2013, A\&A, 553, A130

Fritz, J., Franceschini, A., \& Hatziminaoglou, E. 2006, MNRAS, 366, 767

Fu, H., Cooray, A., Feruglio, C., et al. 2013, Natur, 498, 338

Galametz, M., Kennicutt, R. C., Calzetti, D., et al. 2013, MNRAS, 431, 1956

Gao, Y., Carilli, C. L., Solomon, P. M., \& Vanden Bout, P. A. 2007, ApJL, 660, L93

Gao, Y., \& Solomon, P. M. 2004a, ApJS, 152, 63

Gao, Y., \& Solomon, P. M. 2004b, ApJS, 606, 271

García-Burillo, S., Usero, A., Alonso-Herrero, A., et al. 2012, A\&A, 539, A8

Genzel, R., Tacconi, L. J., Graciá-Carpio, J., et al. 2010, MNRAS, 407, 2091

Gerritsen, J. P. E. 1997, PhD thesis, Rijksuniversiteit Groningen

Graciá-Carpio, J., García-Burillo, S., \& Planesas, P. 2008a, APSS, 313, 331

Graciá-Carpio, J., García-Burillo, S., Planesas, P., Fuente, A., \& Usero, A. 2008b, A\&A, 479, 703

Greve, T. R., Bertoldi, F., Smail, I., et al. 2005, MNRAS, 359, 1165

Greve, T. R., Papadopoulos, P. P., Gao, Y., \& Radford, S. J. E. 2009, ApJ, 692,1432

Griffin, M. J., Abergel, A., Abreu, A., et al. 2010, A\&A, 518, L3

Hailey-Dunsheath, S., Nikola, T., Stacey, G. J., et al. 2008, ApJL, 689, L109 
Harris, A. I., Baker, A. J., Frayer, D. T., et al. 2012, ApJ, 752, 152 Harris, A. I., Baker, A. J., Zonak, S. G., et al., ApJ, 723, 1139 Hezaveh, Y. D., Marrone, D. P., \& Holder, G. P. 2012, ApJ, 761, 20

Hodge, J. A., Carilli, C. L., Walter, F., et al. 2012, ApJ, 760, 11 Inami, H., Armus, L., Charmandaris, V., et al. 2013, ApJ, 777, 16 Iono, D., Wilson, C. D., Yun, M. S., et al. 2009, ApJ, 695, 1537 Ivison, R. J., Papadopoulos, P. P., Smail, I., et al. 2011, MNRAS, 412, 1913 Ivison, R. J., Smail, I., Papadopoulos, P. P., et al. 2010, MNRAS, 404, 198 Ivison, R. J., Swinbank, A. M., Smail, I., et al. 2013, ApJ, 722, 137 Juneau, S., Narayanan, D. T., Moustakas, J., et al. 2009, ApJ, 707, 1217

Kelly, B. C. 2007, ApJ, 665, 1489

Kennicutt, R. C., Jr. 1989, ApJ, 344, 685

Kennicutt, R. C., Jr. 1998, ApJ, 498, 541

Kim, C. G., Ostriker, E. C., \& Kim, W.-T. 2013, ApJ, 776, 1

Knudsen, K. K., Neri, R., Kneib, J.-P., \& van der Werf, P. P. 2009, A\&A, 496, 45

Kovács, A., Chapman, S. C., Dowell, C. D., et al. 2006, ApJ, 650, 592

Krumholz, M., \& Thompson, T. A. 2007, ApJ, 669, 289

Leroy, A., Walter, F., Brinks, E., et al. 2008, AJ, 136, 2782

Leroy, A., Walter, F., Sandstrom, K., et al. 2013, AJ, 146, 19

Lupu, R. E., Scott, K. S., Aretxaga, I., et al. 2012, ApJ, 757, 135

Makiwa, G., Naylor, D. A., Ferlet, M., et al. 2013, ApOpt, 52, 3864

Mao, R. Q., Henkel, C., Schulz, A., et al. 2000, A\&A, 358, 433

Mao, R. Q., Schulz, A., Henkel, C., et al. 2010, ApJ, 724, 1336

Maraston, C. 2005, MNRAS, 362, 799

Meijerink, R., Spaans, M., \& Israel, F. P. 2007, A\&A, 461, 793

Meijerink, R., Spaans, M., Loenen, A. F., \& van der Werf, P. P. 2011, A\&A, 525, A119

Meijerink, R., Kristensen, L. E., Weiß, A., et al. 2013, ApJL, 762, L16

Mendez-Delmestre, K., Blain, A. W., Alexander, D. M., et al. 2007, ApJL, 655, L65

Narayanan, D., Cox, T. J., Shirley, Y., et al. 2008, ApJ, 684, 996

Narayanan, D., Groppi, C. E., Kulesa, C. A., \& Walker, C. K. 2005, ApJ, 630,269

Narayanan, D., \& Krumholz, M. 2014, MNRAS, 442, 1411

Neri, R., Genzel, R., Ivison, R. J., et al. 2003, ApJL, 597, L116

Noll, S., Burgarella, D., Giovannoli, E., et al. 2009, A\&A, 507, 1793

Ostriker, E. C., \& Shetty, R. 2011, ApJ, 731, 41

Panuzzo, P., Rangwala, N., Rykala, A., et al. 2010, A\&A, 518, L37

Papadopoulos, P. P., van der Werf, P. P., Isaak, K., \& Xilouris, E. M. 2012, ApJ, 715,775

Papadopoulos, P. P., Zhang, Z.-Y., Xilouris, E. M., et al. 2014, ApJ, 788, 153

Petric, A. O., Armus, L., Howell, J., et al. 2011, ApJ, 730, 28

Pilbratt, G. L., Riedinger, J. R., Passvogel, T., et al. 2010, A\&A, 518, L1

Planck Collaboration, Ade, P. A. R., Aghanim, N., Armitage-Caplan, C., et al. 2013, A\&A, in press (arXiv:1303.5076)

Rangwala, N., Maloney, P. R., Glenn, J., et al. 2011, ApJ, 743, 94
Riechers, D. A., Bradford, C. M., Clements, D. L., et al. 2013, Natur, 496, 329

Riechers, D. A., Cooray, A., Omont, A., et al. 2011a, ApJL, 733, L12

Riechers, D. A., Hodge, J. A., Walter, F., Carilli, C. L., \& Bertoldi, F. 2011b, ApJL, 739, L31

Riechers, D. A., Walter, F., \& Carilli, C. L. 2007, ApJL, 671, L13

Robson, E. I., Ivison, R. J., Smail, I., et al. 2014, ApJ, 793, 11

Rosenberg, M. J. F., Kazandjian, M. V., van der Werf, P. P., et al. 2014a, A\&A, 564, A126

Rosenberg, M. J. F., Werf, P., Aalto, S., et al. 2014, ApJ, submitted

Sanders, D. B., Mazzarella, J. M., Kim, D.-C., et al. 2003, AJ, 126, 1607

Sanduleak, N. 1969, AJ, 74, 47

Schmidt, M. 1959, ApJ, 129, 243

Schöier, F. L., van der Tak, F. F. S., van Dishoeck, E. F., \& Black, J. H. 2005, A\&A, 432, 369

Schruba, A., Leroy, A. K., Walter, F., et al. 2011, ApJ, 142, 37

Scoville, N. Z., \& Polletta, M. 2001, in ASP Conf. Ser. 249, The Central Kiloparsec of Starbursts and AGN: The La Palma Connection, ed. J. H. Knapen, J. E. Beckman, I. Shlosman, \& T. J. Mahoney (San Francisco, CA: ASP), 591

Serjeant, S. 2012, MNRAS, 424, 2429

Sharon, C. E., Baker, A. J., Harris, A. I., \& Thomson, A. P. 2013, ApJ, 765,6

Shetty, R., \& Ostriker, E. C. 2012, ApJ, 754, 2

Solomon, P. M., Downes, D., Radford, S. J. E., et al. 1992, ApJL, 387, L55

Solomon, P. M., Downes, D., Radford, S. J. E., \& Barrett, J. W. 1997, ApJ, 478, 144

Solomon, P. M., \& Vanden Bout, P. A. 2005, ARA\&A, 43, 677

Stierwalt, S., Armus, L., Charmandaris, V., et al. 2014, ApJ, 790, 21

Swinbank, A. M., Smail, I., Longmore, S., et al. 2010, Natur, 464, 733

Tacconi, L. J., Neri, R., Chapman, S. C., et al. 2006, ApJ, 640, 288

Tacconi, L. J., Neri, R., Genzel, R., et al. 2013, ApJ, 768, 74

Teng, S. H., Brandt, W. N., Harrison, F. A., et al. 2014, ApJ, 785, 19

Thompson, T. A., Quataert, E., \& Murray, N. 2005, ApJ, 630, 167

Thomson, A. P., Ivison, R. J., Smail, I., et al. 2012, MNRAS, 425, 2203

van der Werf, P. P., Isaak, K. G., Meijerink, R., et al. 2010, A\&A, 518, L42

Veilleux, S., Rupke, D. S. N., Kim, D.-C., et al. 2009, ApJS, 182, 628

Walter, F., Decarli, R., Carilli, C. L., et al. 2012, Natur, 486, 233

Wang, J., Zhang, Z., \& Shi, Y. 2011, MNRAS, 416, L21

Ward, J. S., Zmuidzinas, J., Harris, A. I., \& Isaak, K. G. 2003, ApJ, 587, 171

Weiß, A., Ivison, R. J., Downes, D., et al. 2009, ApJL, 705, L45

Wong, M., \& Blitz, L. 2002, ApJ, 569, 157

Wright, E. L., Mather, J. C., Bennett, C. L., et al. 1991, ApJ, 381, 200

Wu, J., Evans, N. J., II, Gao, Y., et al. 2005, ApJL, 635, L173

Wu, J., Evans, N. J., II, Shirley, Y. L., \& Knez, C. 2010, ApJS, 188, 313

Yao, L., Seaquist, E. R., Kuno, N., \& Dunne, L. 2003, ApJ, 588, 771

Zhang, Z.-Y., Gao, Y., Henkel, C., et al. 2014, ApJL, 784, L31 